\title{
Large-Eddy Simulations of Strongly Precipitating, Shallow, Stratocumulus-Topped Boundary Layers
}

\author{
BJORN STEVENS \\ National Center for Atmospheric Research, Boulder, Colorado \\ WiLLIAM R. COTTON \\ Department of Atmospheric Science, Colorado State University, Fort Collins, Colorado \\ GRAHAM FEINGOLD \\ Cooperative Institute for Research in the Atmosphere, Colorado State University, Fort Collins, Colorado \\ Chin-Hoh Moeng \\ National Center for Atmospheric Research, Boulder, Colorado
}

(Manuscript received 20 February 1997, in final form 5 April 1998)

\begin{abstract}
Large-eddy simulations that incorporate a size-resolving representation of cloud water are used to study the effect of heavy drizzle on PBL structure. Simulated surface precipitation rates average about $1 \mathrm{~mm}^{\text {day }}{ }^{-1}$. Heavily drizzling simulations are compared to nondrizzling simulations under two nocturnal PBL regimes—one primarily driven by buoyancy and the other driven equally by buoyancy and shear. Drizzle implies a net latent heating in the cloud that leads to sharp reductions in both entrainment and the production of turbulent kinetic energy by buoyancy (particularly in downdrafts). Drizzle, which evaporates below cloud base, promotes a cooler and moister subcloud layer that further inhibits deep mixing. The cooling and moistening is in quantitative agreement with some observations and is shown to favor the formation of cumuli rising out of the subcloud layer. The cumuli, which are local in space and time, are responsible for most of the heat and moisture transport. They also appear to generate a larger-scale circulation that differs dramatically from the regularity typically found in nonprecipitating stratocumulus. Time-averaged turbulent fluxes of heat and moisture increase in the presence of precipitation, suggesting that drizzle (and drizzle-induced stratification) should not necessarily be taken as a sign of decoupling. Because drizzle primarily affects the vertical distribution of buoyancy, shear production of turbulent kinetic energy mitigates some of the effects described above. Based on large-eddy simulation the authors hypothesize that shallow, well-mixed, radiatively driven stratocumulus cannot persist in the presence of heavy drizzle. In accord with some simpler models, the simulated case with heavy precipitation promotes a reduction in both liquid-water path and entrainment. However, the simulations suggest that timeintegrated cloud fraction may increase as a result of drizzle because thinner precipitating clouds may persist longer if the boundary layer does not deepen as rapidly. These somewhat more complicated dynamics have important implications for a number of hypotheses suggesting that changes in aerosol concentrations, when metabolized by stratocumulus, have a significant effect on climate.
\end{abstract}

\section{Introduction}

In this paper we describe large-eddy simulations (LES) of idealized, nocturnal, stratocumulus-topped, marine PBLs. Our calculations differ from most previous work in that we include a drop-size-resolving representation of cloud microphysical processes, enabling

Corresponding author address: Dr. Bjorn Stevens, Department of Atmospheric Sciences, University of California, Los Angeles, CA 90095 .

E-mail: bstevens@atmos.ucla.edu us to compare calculations with and without drizzle. Our results are used to address a number of outstanding questions about the role of drizzle in the stratocumulustopped marine PBL.

Interest in precipitating stratocumulus is driven partly by the recent recognition that it is more prevalent than once thought (cf. Mason and Howarth 1952; Kraus 1963; Brost et al. 1982a; Nicholls 1984; Nicholls and Leighton 1986; Austin et al. 1995; Bretherton et al. 1995; Boers et al. 1996) and partly by outstanding questions regarding its effect on climate. Currently, a number of intriguing hypotheses state that changes in the atmospheric aerosol may affect the precipitation efficien- 
cy of persistent regions of marine boundary layer cloud, which in turn could alter the structure and radiative properties of these regions. For instance, Albrecht (1989) shows that increased aerosol concentrations may lead to larger cloud fractions as the precipitation efficiency of PBL clouds is reduced. Another idea, related to the suggestion that clouds with insufficient optical depth have unstable steady states (Randall and Suarez 1984), is that PBLs may collapse when precipitation is efficient (Ackerman et al. 1993). Lastly, it has been suggested that steady-state stratocumulus cloud depth is a strictly increasing function of drizzle rate (Wang and Albrecht 1986; Pincus and Baker 1994).

Drizzle is a complicated process involving many scales so it is no surprise that those hypotheses that depend on it are strongly dependent on poorly tested assumptions. Albrecht's result is largely a statement of a simple cloud fraction parameterization. The study by Pincus and Baker neglects the effect of precipitation on PBL vertical structure. Neither model is based on a detailed understanding of how precipitation interacts with PBL turbulence. The model used by Ackerman et al. (1993) is considerably more complicated, but flawed. It neglects leading-order terms when the microphysical model is coupled to the dynamical model and as a result is unable to reproduce rudimentary features of actual stratocumulus-topped PBLs (Stevens et al. 1997). Nonetheless, the above-cited hypotheses are interesting in their own right, and raise some simply stated questions: How does drizzle interact with turbulence in stratocumulus-topped PBLs? Is, as presumed by Pincus and Baker, the impact of drizzle on boundary layer structure not important to the dynamics? Or does heavy drizzle imply smaller cloud fraction and perhaps PBL collapse as suggested in the other studies?

In recalling previous work, we note that while there have been a number of studies of drizzle over the past years, most are interested in questions of a more microphysical nature (e.g., Mason and Howarth 1952; Nicholls 1987; Austin et al. 1995; Feingold et al. 1996; Gerber 1996); nonetheless, some of the early work is of direct relevance to the questions at hand and warrants further discussion. Observational studies show that precipitation is associated with mesoscale fluctuations of order $10 \mathrm{~km}$ in which total-water mixing ratios are elevated by $0.5 \mathrm{~g} \mathrm{~kg}^{-1}$ and temperatures are depressed by about 0.5 K (Paluch and Lenschow 1991; Wang and Wang 1994). Such observations led Paluch and Lenschow to develop a conceptual model in which drizzleinduced stabilization across cloud base helps dry out the cloud layer, and evaporation of drizzle in the subcloud layer promotes the development of a conditionally unstable layer. Cumulus clouds that rise out of this layer further help to break up the upper-cloud layer. Through such a mechanism, drizzle is hypothesized to play a role in the stratocumulus transition to trade cumulus, although subsequent theoretical work suggests that it is not a necessary condition for the transition to occur
(Krueger et al. 1995a; Wyant et al. 1997). A key, but uncorroborated, element of the Paluch and Lenschow model is that drizzle (through decoupling the PBL as initially suggested by Brost et al. 1982b) promotes a drying of the cloud layer, ${ }^{1}$ an idea akin to the suggestion that drizzle might provide an effective means for limiting stratocumulus liquid-water path (LWP) (Nicholls 1987).

Wang and Wang (1994) complemented their observational analyses with a modeling study in which they incorporated a parametric model of drizzle into a onedimensional third-order closure model of PBL turbulence. While they found that drizzle lessened boundary layer turbulent kinetic energy (TKE) (as previously discussed by Ackerman et al. 1993), their approach becomes problematic when increasing drizzle begins to imply more skewed circulations. Moreover the highly parametric nature of their formulation warrants further study of the issue using theoretical models more closely related to first principles. In addition, their work is worth revisiting as it left open the question as to the reason why the TKE is less in the presence of drizzle. Previous studies suggested that the TKE reduction was due to a lessening of the radiative forcing once drizzle thins the cloud layer (Chen and Cotton 1987; Ackerman et al. 1993); Wang and Wang associated it more with a stabilization of the layer due to the redistribution of latent heat.

To summarize, this paper examines how drizzle impacts the turbulent PBL structure in large-eddy simulations of radiatively driven, shallow, nocturnal, stratocumulus-topped PBLs. In contrast to previous studies we use a rather detailed modeling approach and are primarily interested in how the redistribution of latent heat by drizzle affects the growth of the PBL, the turbulent intensity, and the fluxes of heat and moisture. Such questions are of direct relevance to a number of studies that have postulated the atmospheric aerosol, by modifying the efficiency of precipitation in PBL clouds, may modify the earth's radiation budget. Our methodology is outlined in more detail in section 2 ; our calculations and a discussion of these calculations follow in subsequent sections.

\section{Approach}

\section{a. Formulation}

The model is more completely described in previous work (Stevens et al. 1996, hereafter SFCW) and is similar in spirit to that described by Kogan et al. (1995) although we currently treat aerosol-cloud drop interactions in a less sophisticated manner. Our model solves

\footnotetext{
${ }^{1}$ Here decoupling can be taken in the weak sense, i.e., a local minimum in the fluxes at some $z<z_{i}$. Further discussion of this issue is postponed until section $5 \mathrm{a}$.
} 
TABLE 1. Initial conditions for all calculations. Values of the geostrophic meridional wind $v_{g}$ for the different simulations are given in Table 2.

\begin{tabular}{rcccc}
\hline \hline $\begin{array}{c}\text { Height } \\
(\mathrm{m})\end{array}$ & $\theta(\mathrm{K})$ & $\begin{array}{c}q_{t} \\
\left(\mathrm{~g} \mathrm{~kg}^{-1}\right)\end{array}$ & $\begin{array}{c}u \\
\left(\mathrm{~m} \mathrm{~s}^{-1}\right)\end{array}$ & $v\left(\mathrm{~m} \mathrm{~s}^{-1}\right)$ \\
\hline 0.0 & 288.000 & 10.200 & -0.7 & $v_{g}$ \\
662.5 & 288.000 & 10.200 & -0.7 & $v_{g}$ \\
712.5 & 293.500 & 9.100 & -2.0 & $v_{g}$ \\
2500.0 & 304.225 & 4.095 & -2.0 & $v_{g}$ \\
\hline
\end{tabular}

prognostic equations for up to 57 variables over a threedimensional grid of $64 \times 64 \times 48$ points. These variables are the three components of velocity, the perturbation Exner function, liquid-water potential temperature $\theta_{l}$, total-water mixing ratio $q_{t}, 50$ additional scalars that describe the liquid-water size distribution function, and one scalar that helps describe the supersaturation field. For the shallow flows (i.e., the depth of the flow is much less than a density scale height) to be described here, the model approximately solves an anelastic continuity equation. Changes to the model include the addition of stochastic collection and drop sedimentation to the detailed microphysical calculations (as described by Feingold et al. 1996); other changes (see appendix for more details) include a slight modification to the subgrid model, the inclusion of ventilative effects in the condensation-evaporation equations, and the replacement of the emissivity-based radiation scheme with a very simplified representation of nocturnal, infrared, radiative forcing.

Our calculations are initialized using a piecewise-linear interpolation of the sounding in Table 1, which is based on idealizations of measurements taken from ASTEX the Atlantic Stratocumulus Transition Experiment) flight 219 (P. G. Duynkerke 1996, personal communication). At the initial time a pseudorandom perturbation, $\delta \theta_{l} \in(-0.1,0.1)$, whose mean vanishes on each level, is applied for $z \leq 662.5 \mathrm{~m}$. Constants in the radiation model allow for up to $74 \mathrm{~W} \mathrm{~m}^{-2}$ to be extracted from the PBL (see the appendix for more details). For the simulations that use the detailed microphysical model, it is assumed (for the purposes of drop activation) that ammonium sulfate aerosol number mixing ratios are fixed in time at $25 \mathrm{mg}^{-1}$ and can be described by a single lognormal distribution function with parameters $\left(D_{g}, \sigma_{d}\right)=(0.2 \mu \mathrm{m}, 1.5)$. Such a large mode radius forces drop concentrations to be nearly equal to the aerosol concentrations since the former are more readily accessible given the range of supersaturation typically produced, hence these aerosols will be hereafter referred to as $\mathrm{CCN}$ without regard to a specific activation supersaturation. In all simulations large-scale divergence is fixed at $5 \times 10^{-6} \mathrm{~s}^{-1}$, and the grid is stretched (with grid-stretch ratio of $10 \%$ ) between $900 \mathrm{~m}$ and the model top near $1500 \mathrm{~m}$. A Rayleigh friction damping layer is applied in the upper $400 \mathrm{~m}$ (five layers) of the domain with a damping timescale of $60 \mathrm{~s}$. Lateral boundary
TABLE 2. Name, description, and discretization of primary numerical experiments. The value of the geostrophic zonal wind $v_{g}$ is -2 $\mathrm{m} \mathrm{s}^{-1}$. Lengths in meters, time in seconds in the experiment names, B refers to buoyancy forcing, $\mathrm{S}$ refers to shear forcing, ND means no drizzle, and HD means heavy drizzle. (For example, experiment HDBS is the heavy drizzle simulation with buoyancy and shear forcing.)

\begin{tabular}{lccc}
\hline \hline $\begin{array}{c}\text { Experiment } \\
\text { name }\end{array}$ & $\begin{array}{c}\text { Bin } \\
\text { microphysics }\end{array}$ & $\begin{array}{c}v_{g} \\
\left(\mathrm{~m} \mathrm{~s}^{-1}\right)\end{array}$ & $(\Delta x, \Delta y, \Delta z, \Delta t)$ \\
\hline NDBS & No & -10 & $(50,50,25,2 \mathrm{~s})$ \\
NDB & No & 2 & $(50,50,25,2 \mathrm{~s})$ \\
HDBS & Yes & -10 & $(60,60,30,2 \mathrm{~s})$ \\
HDB & Yes & 2 & $(60,60,30,2 \mathrm{~s})$ \\
\hline
\end{tabular}

conditions are periodic. The lower boundary has the characteristics of water held at a fixed temperature of $290.4 \mathrm{~K}$ and surface fluxes are derived on the basis of similarity theory (see SFCW). The numerics are essentially unchanged from what is described in SFCW.

To conserve resources, integrations without detailed microphysics are used as a proxy for integrations with a very large concentration of CCN. These nondrizzling cases diagnose the liquid water and temperature by assuming that cloudy air is just saturated and that all liquid water is in the form of cloud drops whose fall velocity is negligible. This approach eliminates the need to prognosticate cloud liquid water. Two-dimensional simulations show that calculations based on such a simplified representation of microphysical processes (which is typically used in studies of stratocumulus without precipitation) approximate well calculations with very many available condensation sites (Stevens 1996). Many of the questions raised in the introduction focus on the effect of varying $\mathrm{CCN}$ concentrations on the precipitation efficiency of stratocumulus; our two-dimensional calculations show that the effect of a graduated change in CCN concentrations is well illustrated by only considering the extreme states of heavy and no drizzle as are considered here.

Lastly, to develop a somewhat broader view of the role of drizzle, simulations with heavy and no drizzle are performed in two PBL regimes-leading to a total of four simulations. The first PBL regime (experiments NDB and HDB in Table 2) has a light geostrophic wind and is mainly buoyancy driven. The second regime has a stronger geostrophic wind and receives approximately equal contributions to TKE production from buoyancy and shear (experiments NDBS and HDBS in Table 2). The nondrizzling (ND) integrations use a slightly more refined spatial resolution but are insensitive to this change; sensitivity studies with $(\Delta x=\Delta y, \Delta z)$ varied by nearly a factor of 2 showed relatively little change in the results. ${ }^{2}$

\footnotetext{
${ }^{2}$ Subsequent simulations indicate that this is in part because independent sensitivities of opposite sign to refinements in horizontal and vertical resolution largely compensate for one another. However, even these independent sensitivities are not nearly as strong as those associated with heavy drizzle.
} 


\section{b. Assumptions}

Ultimately we would like to extend our interpretation of our calculations to physical reality, if only in the form of a refined hypothesis. In so doing, one can only benefit from a clear articulation of what we believe to be the major assumptions/weaknesses underlying our approach. Such a discussion of underlying assumptions serves the added benefit of defining fruitful avenues for future investigation.

\section{1) Cloud dRop ACTIVATION}

In this regard our chief assumption is that a very crude representation of the interaction of the atmospheric aerosol and cloud drops is sufficient to elucidate the effects of precipitation on PBL structure. Our aerosol model assumes that the number of activated drops at any time is given by the difference between the number of $\mathrm{CCN}$ that would activate given the ambient supersaturation and the number of existing drops (SFCW). Unfortunately the use of such a procedure, which is well justified for the activation-condensation problem, no longer holds for precipitating flows in which collection is active and cloud condensation nuclei $(\mathrm{CCN})$ are not conserved.

The use of such a simple scheme is justified both on practical grounds and by the fact that we are more interested in how the redistribution of latent heat by drizzle affects PBL turbulence, and less interested in the detailed microphysical evolution of the precipitating layer. A realistic treatment of the aerosol budget would introduce another timescale into the problem, which would complicate our analysis and greatly increase the computational cost of the calculation.

\section{2) MICROPHYSICAL PROCESSES}

Similarly to what has been described by Feingold et al. (1996) the two moment method (Tzivion et al. 1987), with the collection kernels described by Long (1974), is used. Long's kernel is known to accelerate coalescence growth, and indeed two-dimensional simulations (Stevens 1996) indicate that it increases precipitation production by $30 \%$ relative to the kernel compiled by Hall (1980). This enhancement in precipitation production is comparable to what is achieved by increasing the total-water mixing ratio in the sounding by $1 \%$ (Stevens 1996). Thus, relative to other uncertainties and approximations (i.e., our neglect of the aerosol budget and that the microphysical calculations in our model do not account for processes on scales smaller than the grid scale — which is known to cause problems at cloud edges), we believe that errors introduced by using Long's kernel are tolerable.

Despite that we have made an extraordinary effort to ensure that microphysical processes are well represented by the model, it is ultimately very difficult to assess the degree to which the model produces reasonable precip- itation rates for the given thermodynamical and dynamical conditions, in part because previous observational studies have not been designed with the objective of constraining such calculations. Tests show that the model realistically responds to reductions in aerosol concentrations by progressively reducing the rate of precipitation production (Stevens 1996). Furthermore, when compared to published observations (e.g., Bretherton et al. 1995; Paluch and Lenschow 1991; Wang and Wang 1994), calculated surface precipitation rates are reasonable, as are precipitation-induced anomalies in subcloud temperatures and vapor-mixing ratios.

\section{3) ENTRAinMENT}

How well does the model represent entrainment? Although not immediately apparent, this is an important question because much of the change in the dynamics between the precipitating and nonprecipitating calculations is mediated by altered entrainment rates. Recent studies are ambiguous as to the effect of refinements of the horizontal mesh on entrainment, although it is becoming increasingly clear that entrainment rates decrease as the vertical mesh is refined. Tests with the nonprecipitating version of this case show that entrainment rates are sensitive to changes between a 5- and 3$\mathrm{m}$ vertical mesh. Thus it seems clear that the resolution used here is too coarse to make detailed quantitative statements about the nature of stratocumulus topped PBLs, and we are forced to assume that despite the sensitivity of calculated entrainment rates to resolution, the sensitivity of the model to precipitation, based on physically sound principles, will not be qualitatively affected by this shortcoming.

\section{c. Complementary approaches}

Given the nature of our questions and the limitations addressed above, one is justified in wondering why we use such a complicated microphysical representation of clouds. The drop-size-resolving model increases the computational cost by an order of magnitude and makes it difficult to do many sensitivity studies. Are its benefits worth the additional cost? When this study was initiated little was known about precipitation development in stratocumulus; most models used modified bulk microphysical parameterizations borrowed from simulations of deep convection (Chen and Cotton 1987; Wang and Wang 1994). At the outset the authors believed it to be important to properly simulate the development of precipitation on scales on the order of the cloud depth. This combined with our lack of confidence in existent microphysical parameterizations (i.e., particularly their crude representations of collection and the size sorting of drops within the cloud, e.g., Feingold et al. 1996) further encouraged our choice to use the detailed model. In retrospect, some of our questions might have been better studied using a simpler 

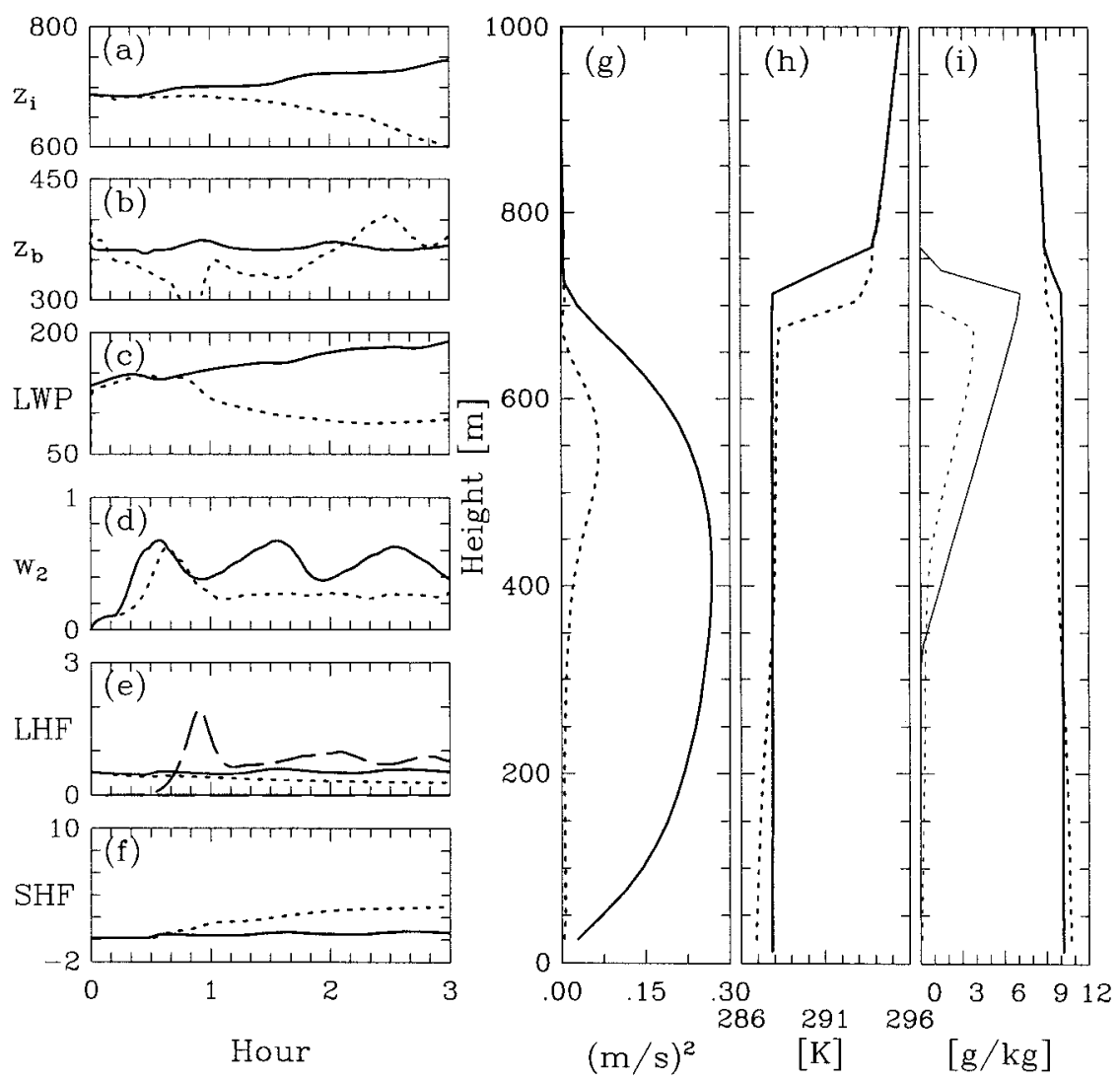

FIG. 1. Time series and time averages over third hour from experiment NDB (solid lines) and HDB (dotted lines): (a) Inversion height measured by average level of $q_{t}=9.65 \mathrm{~g} \mathrm{~kg}^{-1}$ contour at a given time; (b) cloud-base height as indicated by level of $q_{l}=0.01 \mathrm{~g} \mathrm{~kg}^{-1}$ contour; (c) vertically integrated cloud water $\left(\mathrm{g} \mathrm{m}^{-2}\right)$; (d) maximum value of $\overline{w^{\prime} w^{\prime}}$ at each time; (e) latent heat flux $\left(\mathrm{mm} \mathrm{day}^{-1}\right)$ (drizzle flux given by dashed line); (f) sensible heat flux ( $\left.\mathrm{W} \mathrm{m}^{-2}\right)$; $(\mathrm{g})$ $\overline{w^{\prime} w^{\prime}}\left(\mathrm{m}^{2} \mathrm{~s}^{-2}\right)$; (h) $\theta_{l}(\mathrm{~K})$; and (i) $10 q_{l}$ and $q_{t}\left(\mathrm{~g} \mathrm{~kg}^{-1}\right)$.

microphysical model, thereby allowing finer spatial resolution or larger domains. Indeed some of us have recently developed a model with just this purpose in mind (Feingold et al. 1998). Nevertheless, the present analysis (based on simulations performed using the drop-size-resolving model) does illustrate some interesting dynamics and raises many questions worthy of further study.

While we believe that further progress can be made by considering simplified microphysical models integrated over a finer numerical mesh, more detailed calculations of microphysical-aerosol interactions are also very much of interest-if only to help constrain the simplified microphysical models. However, because the microphysical development of the cloud is intimately coupled to the turbulent structure of the PBL (Feingold et al. 1996) and because the latter is so strongly dependent on properly representing cloud-top processes (something limited by available computer resources), realistic microphysical calculations shall remain a challenging problem for some time to come.

\section{Results}

\section{a. Time evolution and mean state}

Selected time series output and mean profiles-averaged over the third hour, from each of the four integrations-are plotted in Figs. 1 and 2. Many of the effects of drizzle are well illustrated by these plots. In both PBL regimes, drizzle is associated with substantially smaller values of $\overline{w^{\prime} w^{\prime}}$ (panel g), cooled and moistened subcloud layers with significantly more stability across the mean cloud base (panels $h$ and i), less entrainment (panel a), and less liquid water (panels c and i). The cooling and moistening of the subcloud layer is consistent with what is observed in stratocumulustopped PBLs precipitating at about the same rate (Wang and Wang 1994). The reduction in turbulent kinetic energy is also evident in the time series of the maximum value of the layer-averaged vertical velocity variance (i.e., $w_{2}$ in panel d). Drizzle rates are about $1 \mathrm{~mm} \mathrm{day}^{-1}$ in both of the precipitating simulations, although they are slightly higher when large mean winds help ventilate 

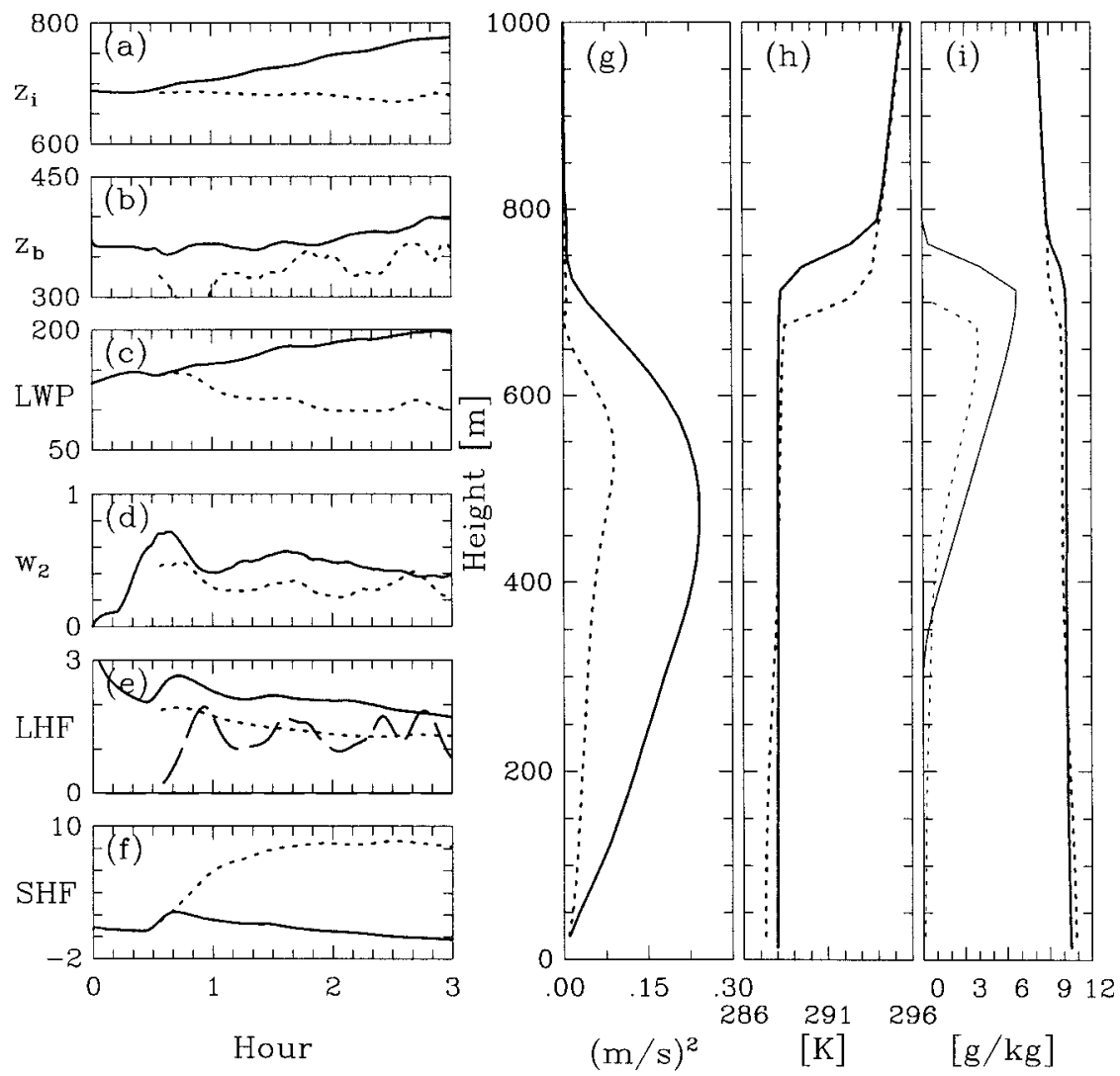

$[\mathrm{K}]$

FIG. 2. As in Fig. 1, but for experiments NDBS and HDBS.

the surface (panel e). In response to the cooling and moistening of the subcloud layer, surface sensible heat fluxes are increased (panel f), and surface latent heat fluxes are reduced (panel e). Cooling in the lowest layer leads to larger exchange coefficients in the surface flux formalism, which mitigates the reduction in surface latent heat fluxes due to the low-level moistening. Overall, the sink of moisture due to drizzle dominates the effective moisture sink associated with reduced surface latent heat fluxes, while differences in the surface sensible heat flux are largely responsible for the differences in the surface buoyancy flux.

Irrespective of drizzle, shear (because it tends to be associated with stronger mean winds) helps ventilate the surface. The integrations with shear also tend to deepen the boundary layer more rapidly then the integrations without shear. In the nondrizzling integrations LWP increases with more entrainment, so experiment NDBS has larger values of LWP than does experiment NDB. Shear also has a significant impact on the shape of the profile of the vertical velocity variance. Because our purpose is to understand how drizzle impacts boundary layer evolution in two different PBL regimes, further discussion of the effects of shear will be limited to its effect on the response of the boundary layer to heavy drizzle.

\section{b. Flow visualization}

Snapshots of the flow augment the mean statistics. Here, we compare snapshots of precipitating and nonprecipitating simulations of the buoyancy-driven PBL; results from the buoyancy and shear regime are less dramatic but similar. Although drizzle leads to reductions in the mean LWP of about a factor of 2 (Fig. 1c), the spatial variance in LWP is increased; for example, local maxima in LWP are considerably larger in the presence of drizzle (cf. panel a in Figs. 3 and 4). The heavily precipitating PBLs also tend to be dominated by fewer circulations, which may be more intense than in the nonprecipitating case but more local in time and space. Hence, the reduction in $\overline{w^{\prime} w^{\prime}}$ with the onset of heavy precipitation better reflects the intermittency in the turbulent circulations than it does their strength.

The convective circulations associated with the heavily precipitating calculations are dominated by updrafts, and are much more "cumulus-like" (cf. Figs. 3b and $4 \mathrm{~b}$ as well as the contoured velocity field in Figs. 5 and 6.). In the precipitating integrations, up- and downdrafts no longer form couplets with commensurate strength and similar levels of cloud base. Instead, updrafts are moister and balanced by a larger region of gentle, dry, downward motion. Because for a fixed mass flux, the 

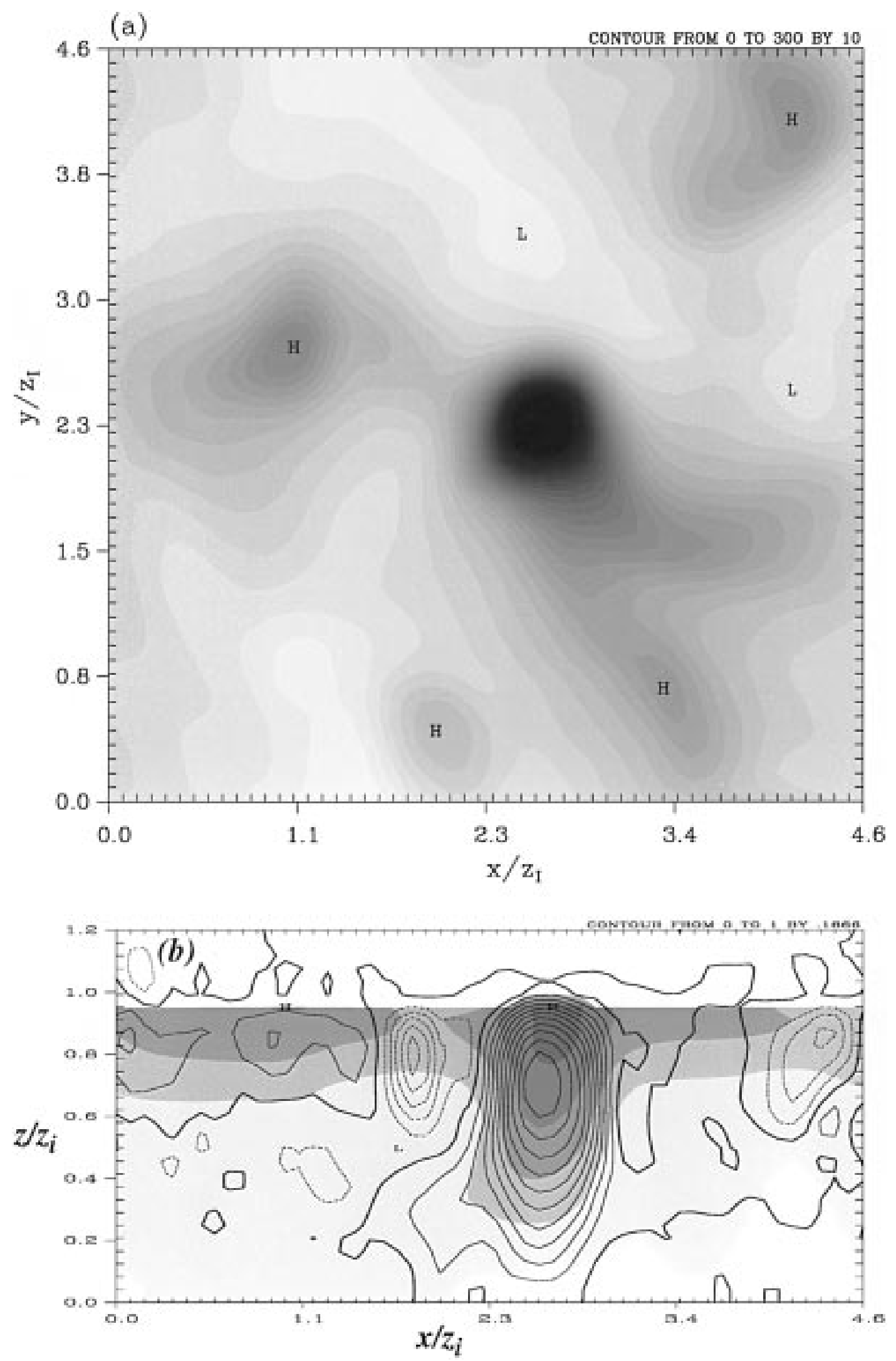

FIG. 3. Snapshots of HDB at $10800 \mathrm{~s}$ : (a) vertically integrated liquid water $\left(\mathrm{g} \mathrm{m}^{-2}\right)$; (b) vertical velocity (contours every $0.1 \mathrm{~m} \mathrm{~s}^{-1}$, zero contour thick, negative velocities dashed), and liquid water (shaded). In (b), contour interval at top of plot refers to shading interval. 

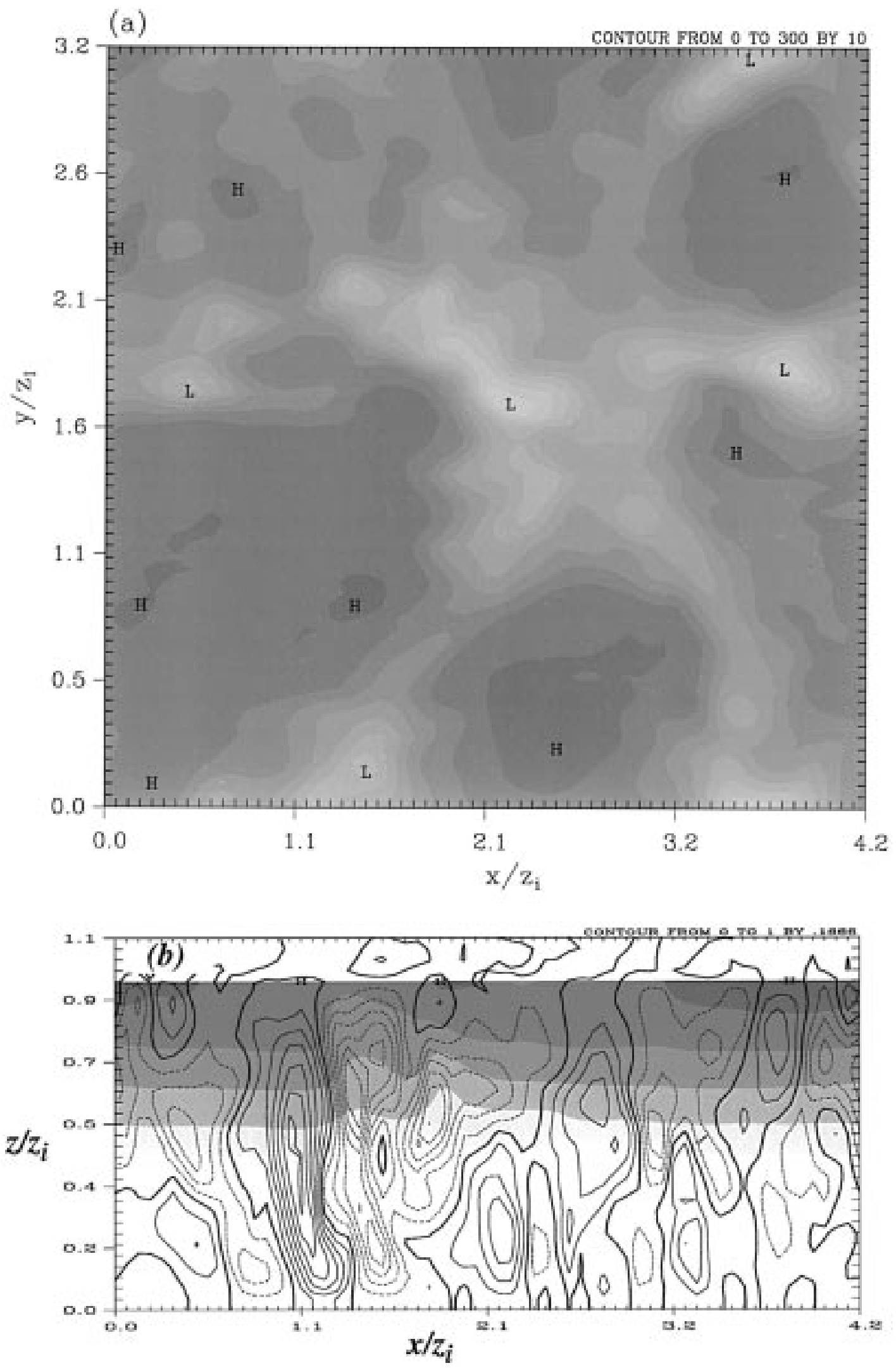

FIG. 4. As in Fig. 3, but for experiment NDB. 

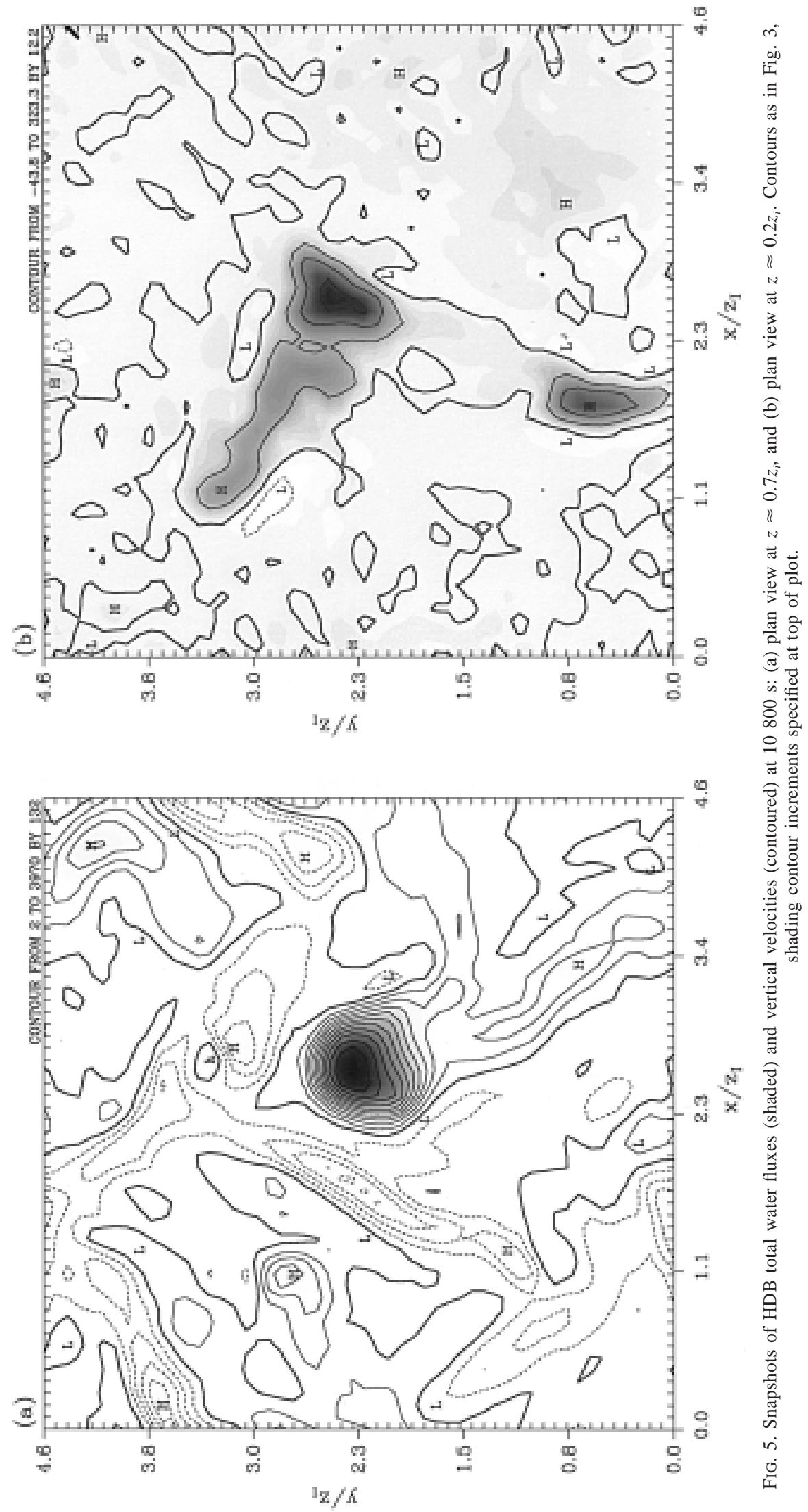

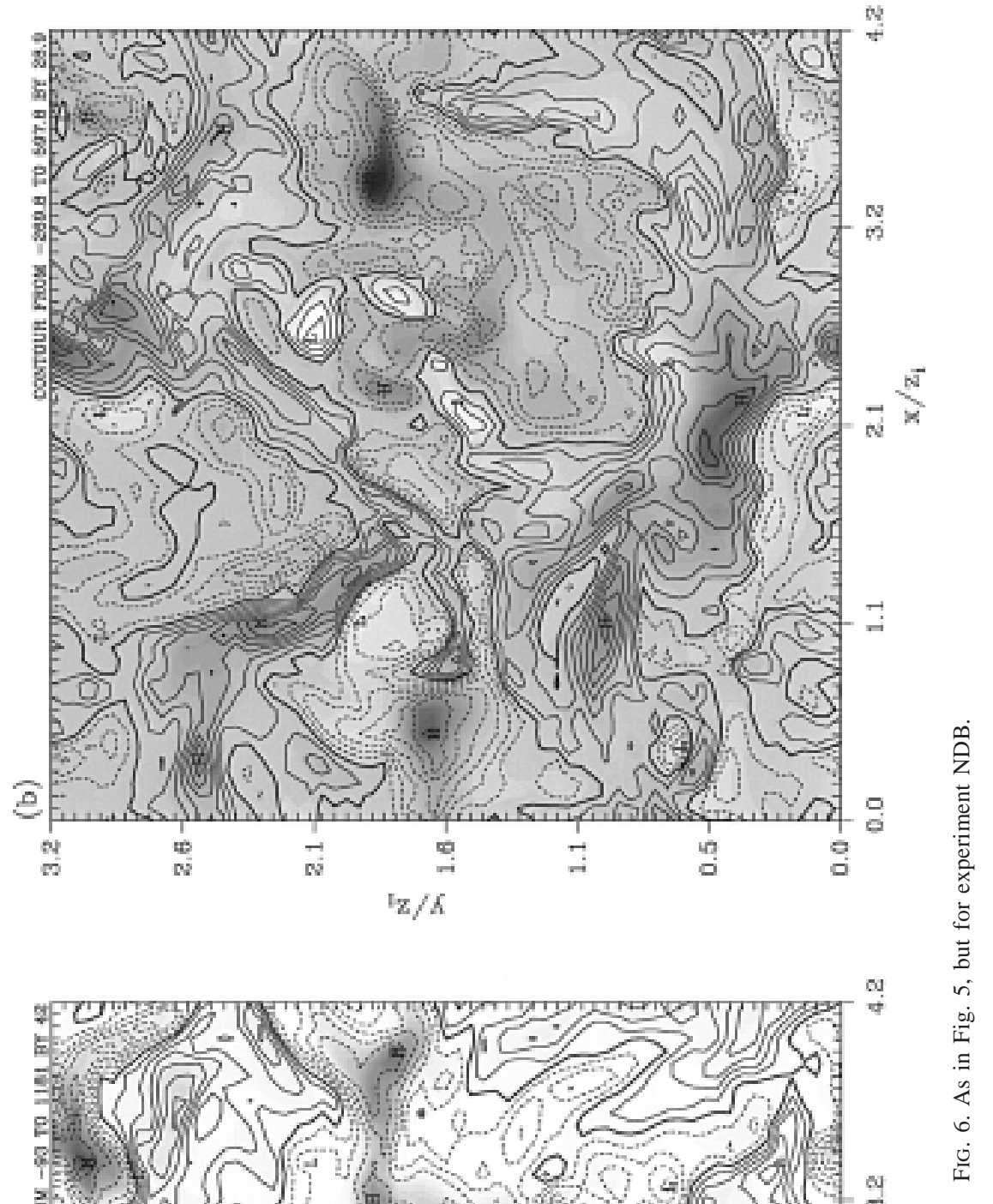
stabilizing effect of a dry, compensating, downward motion is inversely proportional to the downdraft area, this type of circulation favors updrafts on smaller scales (Bjerknes 1938). The skewness $\mathcal{S}_{w}$ of the vertical velocity field is the ratio of third moment to the threehalves power of the second moment of $w$. In the absence of precipitation $\left|S_{w}\right|<0.5$, but in the heavily precipitating integrations $\mathcal{S}_{w} \approx 2.5$. Such a large value of skewness is consistent with the snapshots (strong updrafts covering a small area dominate the circulation) and is similar to what is observed in cumulus-coupled PBLs (De Roode and Duynkerke 1996). The more cumuluslike dynamics are also evident in snapshots of total moisture fluxes superimposed on snapshots of the vertical velocity field (see Figs. 5 and 6 and note the scale change in contours of water flux). Precipitation helps break the transport symmetry evident in the nonprecipitating integrations. Whereas in the absence of precipitation up- and downdrafts contributed equally to the net turbulent flux of moisture; when precipitation is heavy, the turbulent flux is dominated by updrafts.

One disconcerting aspect of the simulations is that there is only one dominant updraft in simulation HDB. In this experiment, the largest scales implied by the physics may be larger than the domain allows. This type of heuristic evidence for upscale growth warrants further study using larger domains. Another disconcerting aspect of the simulations is the lack of structure near cloud top, some of which is a reflection of the contour algorithms. In nature, one tends to observe considerably more cloud-top structure, much of which is probably related to mesoscale variability in both the inversion strength and divergence. However, a sizable fraction may also be due to PBL processes. For instance, a cursory examination of radar data from ASTEX suggests that cloud tops are often higher above regions of active precipitation; a similar effect has also been noted by Jones (1951). It is unclear whether this is because the deeper, more vigorous clouds favor the formation of precipitation, or because precipitating clouds favor larger penetrations into the inversion. Regardless, such a process is not particularly evident in our snapshots, where only a minimal perturbation in cloud-top height can be associated with the vigorous precipitating cumulus elements. Clearly, such issues merit more systematic study.

\section{c. Budgets}

When examining fluxes, it is worthwhile to consider the sum of all fluxes that contribute to the evolution of a particular variable. Hence, in addition to kinematic fluxes (i.e., the resolved and subgrid turbulent fluxes, which are combined below into one term), the superpositions of certain fluxes are also interesting to examine. In particular, we can define two fluxes,

$$
F_{Q}=\rho_{0} L \overline{w^{\prime} q_{t}^{\prime}}+\bar{F}_{\mathrm{drz}}
$$

where

$$
\begin{gathered}
\bar{F}_{\mathrm{drz}}=\rho_{0} L \sum_{k=1}^{25} U_{\infty}\left(\bar{D}_{k}\right) \bar{q}_{l}\left(\bar{D}_{k}\right), \\
F_{\Theta}=\rho_{0} c_{p} \overline{w^{\prime} \theta_{l}^{\prime}}-\bar{F}_{\mathrm{drz}}+\bar{F}_{\mathrm{rad}},
\end{gathered}
$$

whose divergence represents the sole contribution to the evolution of $\overline{q_{t}}$ and $\overline{\theta_{l}}$, respectively. In the drizzle flux term $F_{\mathrm{drz}}$ the value of the terminal velocity $U_{\infty}$ is related to the predicted mean diameter $\bar{D}_{k}$ within each size interval following Gunn and Kinzer (1949). The radiative flux $\bar{F}_{\text {rad }}$ is given by Eq. (6). Overbars represent spatial and time averages over the third hour of the simulation. Fluxes are calculated "on the fly," by extracting them directly from the relevant model algorithm every $30 \mathrm{~s}$ (15 time steps) over a period of an hour. This procedure guarantees that meaningful and representative averages are generated.

If $F_{Q}(z)$ and $F_{\Theta}(z)$ are linear, then their second derivatives vanish, implying that the shape of the layeraveraged profiles of $q_{t}$ and $\theta_{l}$ are not changing with time. This is often referred to as a quasi-steady state. Only the HDB simulation deviates substantially from such a state (see Figs. 7a and 8a) and then only in the moisture budget. A closer analysis of this budget (Fig. 7a) shows that $F_{\mathrm{drz}}$ is commensurate with the net turbulence flux in the cloud layer, but dominates nearer the surface. As a result, layers below $0.25 z_{i}$ are moistening at the expense of the layers above, which are drying at a rate of about $0.05 \mathrm{~g} \mathrm{~kg}^{-1} \mathrm{~h}^{-1}$. This is not the case in integration HDBS, where shear generation of turbulence at low levels helps ventilate the subcloud layer and achieve a quasi-steady state on short timescales. The mean total-water mixing ratio in the cloud layer is about $0.25 \mathrm{~g} \mathrm{~kg}^{-1}$ less (see Figs. 1i and 2i) when the clouds drizzle. To dry a 300-m layer by this amount over two hours requires that $F_{Q}$ change by nearly $40 \mathrm{~W} \mathrm{~m}^{-2}$ more (over the layer) in the drizzling integrations than it does in the nonprecipitating integrations. Interestingly, during the third hour, the gradients in $F_{Q}$ in the cloud layer are about the same for simulations HDBS and NDBS. Even in the absence of shear, the cloud layer is not drying as rapidly as one might expect given the strength of the drizzle flux. Precipitation does lead to significant drying over the upper portion of the cloud layer. However, this drying is largely compensated for by enhanced turbulent fluxes of moisture from the subcloud layer. Recalling the significant reductions in $\overline{w^{\prime} w^{\prime}}$ associated with drizzle motivates the interpretation of this result as an indication that the circulations more efficiently transport moisture in the drizzling-cumulus coupled PBL. Nicholls and Leighton $(1986,448)$ actually observed a similar response in nature, as they note that, "cases with large liquid water fluxes also tend to be those with large rainfall rates."

Drizzle also induces turbulence to work more effi- 

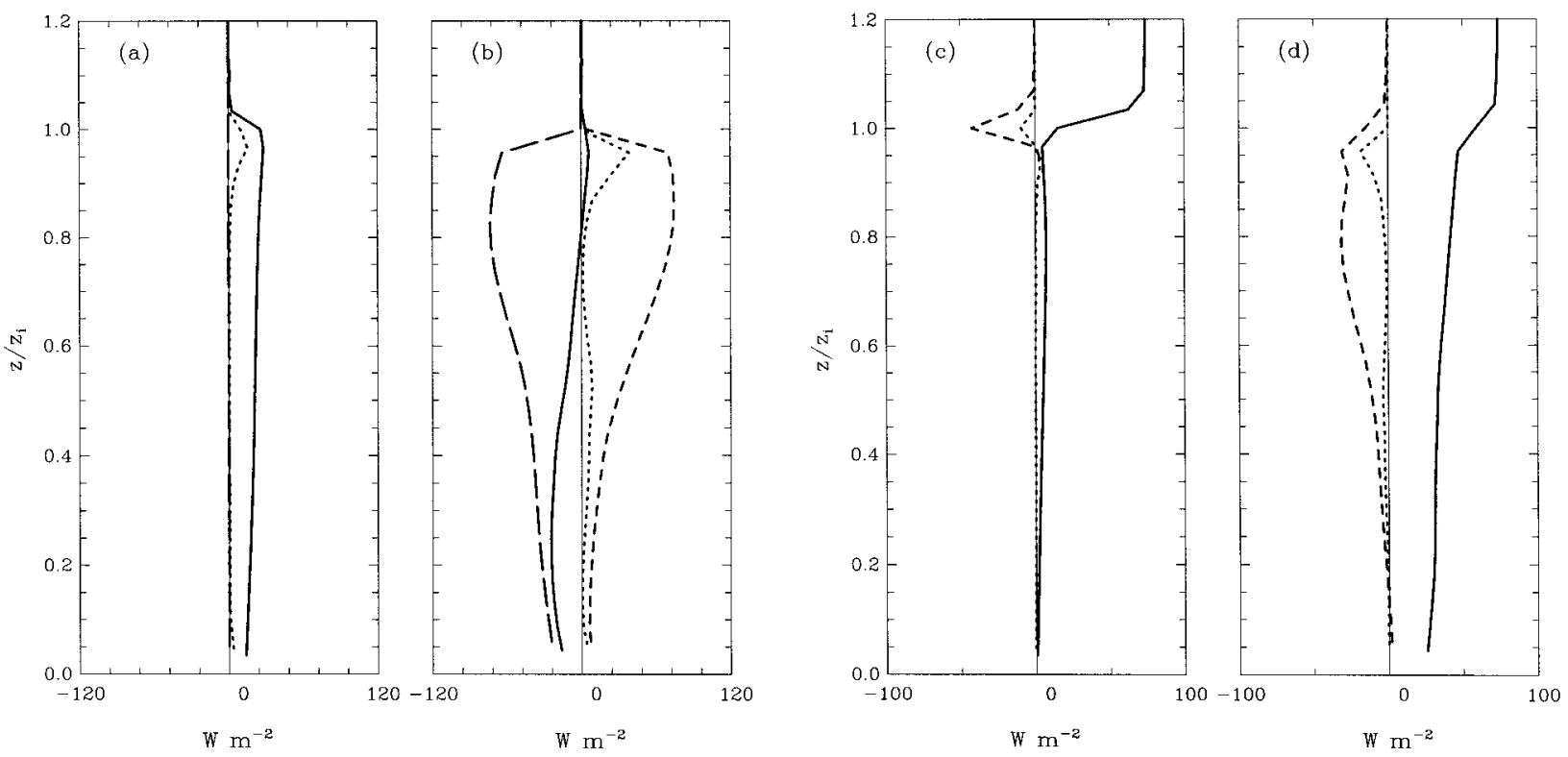

Fig. 7. Second-order statistics averaged over third hour for experiments: (b) and (d) HDB and (a) and (c) NDB. (a) and (b): Total water budget (W m ${ }^{-2}$ ) - total turbulent flux (short dash), subgrid contribution (dotted), $\bar{F}_{\mathrm{drz}}$ (long dash), and $\bar{F}_{Q}$ (solid). (c) and (d): $\theta_{l}$ budget (W $\mathrm{m}^{-2}$ )— total turbulent flux (short dash), subgrid contribution (dotted), and $\bar{F}_{\Theta}$ (solid).

ciently from the perspective of the $\theta_{l}$ budget; that is, turbulent fluxes of $\theta_{l}$ are larger when drizzle is active, even though the resolved-scale turbulent kinetic energy tends to be smaller. In the nonprecipitating integrations, $\overline{w^{\prime} \theta_{l}^{\prime}}$ is set by the entrainment rate for a fixed radiative forcing. In the weakly entraining NDB simulation, $\overline{w^{\prime} \theta_{l}^{\prime}}$ increases slowly with height, implying cooling. When shear contributes to TKE production, entrainment increases and $\overline{w^{\prime} \theta_{l}^{\prime}}$ decreases with height. Precipitation affects these relations. When drizzle is heavy, $\overline{w^{\prime} \theta_{l}^{\prime}}$ decreases more rapidly with height through the subcloud layer and into the middle of the cloud layer. This is where the drizzle flux divergence is largest, indicating that turbulence tends to compensate for the heating/cooling pattern in $F_{\Theta}$. Because the reduction in entrainment warming is not completely compensated for by the cloud-top warming due to drizzle production (even though the turbulent transport of $\theta_{l}$ becomes more efficient), the precipitating solutions tend to $\operatorname{cool}\left(F_{\Theta}\right.$ increases more rapidly with height) at a greater rate than their nondrizzling counterparts.

Budgets of TKE (Figs. 9 and 10) reaffirm earlier arguments and clearly indicate that TKE production decreases when drizzle is active. Wang and Wang (1994) reached similar conclusions, although where we see larger changes in the buoyancy production of TKE in the cloud layer, they saw larger changes in the subcloud layer. Precipitation primarily affects the buoyancy term in the TKE budget, but because the surface friction velocity depends on surface stability, the shear production of TKE may also be modified as drizzle changes the stability characteristics of the surface layer. In both precipitating simulations, the shape of the buoyancy pro- duction profile also changes; below cloud base it is nearer zero and it increases more rapidly toward cloud top. In simulations HDBS and NDBS, shear production is confined to the subcloud layer, but when integrated over the entire boundary layer, it contributes approximately as much as the vertically integrated buoyancy term. In addition to producing more TKE near the surface, shear is associated with strong mean winds that help ventilate the surface. Both of these factors lead to the HDBS simulation being better coupled (i.e., greater mid-PBL fluxes and less evidence of a flux minimum in the PBL interior) than its counterpart with weak shear. Hence, even though drizzle primarily effects buoyancy production of TKE, when considering how precipitation might affect the evolution of stratocumulus layers, it is important to include the effect of shear.

To better understand how drizzle impacts the production of TKE, it helps to think about the buoyancy production of TKE in more detail. In the model the buoyancy variable is the virtual potential temperature,

$$
\theta_{v}=\theta\left(1+0.61 r_{v}-r_{l}\right)
$$

the buoyancy production term in the TKE budget is $\theta_{0}^{-1} g \overline{w^{\prime}} \theta_{v}^{\prime}$. Contributions to this term are plotted in Fig. 11 , as are the relative contributions from up- and downdrafts. A comparison between the simulations reveals some important differences. In the nonprecipitating solution, downdrafts have approximately the same saturation pressure level as updrafts but contribute preferentially to the buoyancy production of TKE. In the precipitating solution, downdrafts are positively buoyant below their level of cloud base (which means that kinetic energy is being converted to potential energy and the 

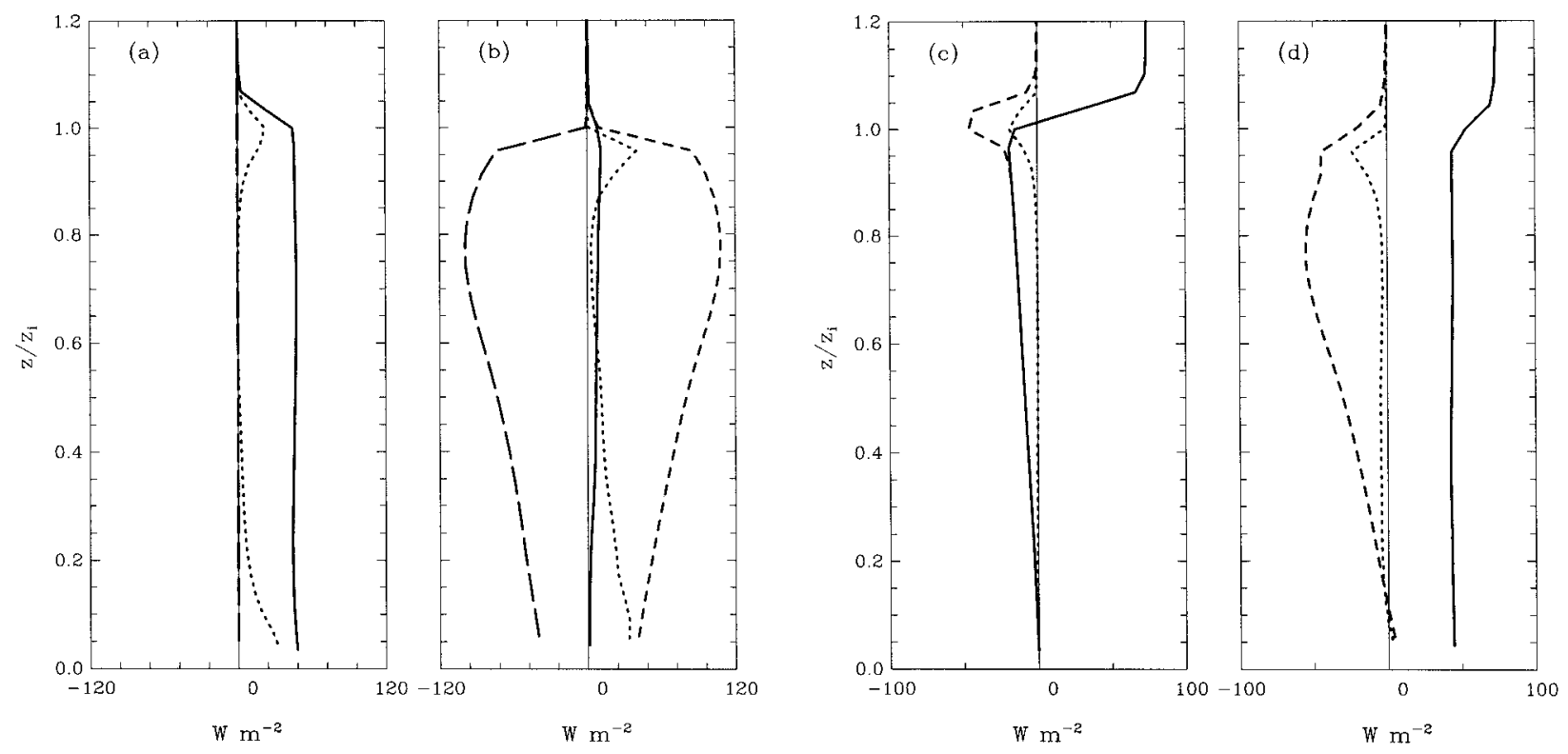

FIG. 8. As in Fig. 7 but for experiments HDBS and NDBS.
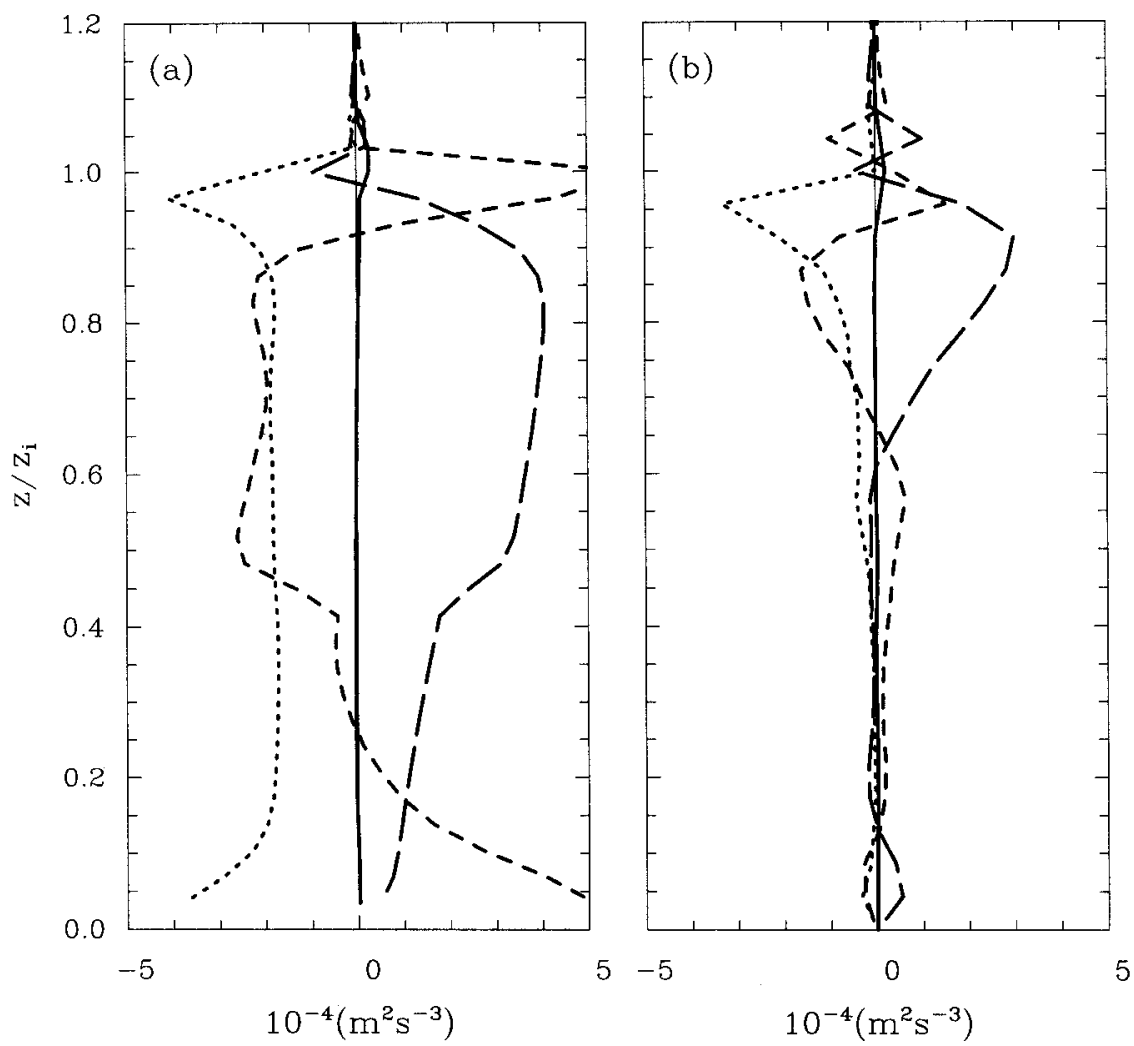

FIG. 9. TKE budgets: (a) experiment NDB and (b) experiment HDB. Shear production (solid), buoyancy production (long-dashed), transport (short-dashed), and dissipation (dotted). 

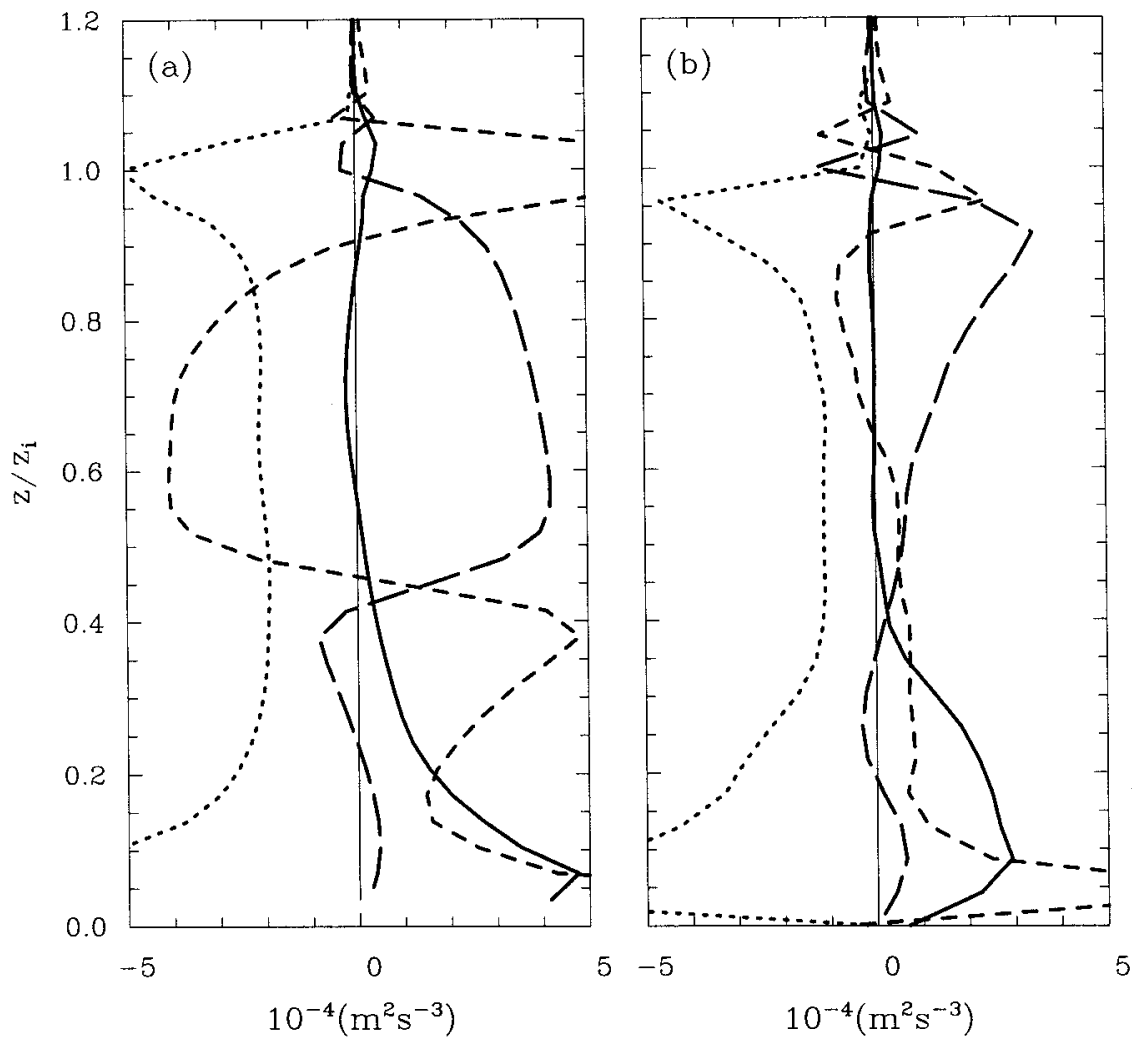

FIG. 10. As in Fig. 9, except for experiments NDBS and HDBS, respectively.

buoyancy production term is negative); thus, much of the kinetic energy generated by radiative cooling at cloud top is used to do work against the stratification. Because the net buoyancy production in the TKE budget is just the superposition of the up- and downdraft components, most of the change in the buoyancy flux due to the changing contribution within downdrafts. Despite that the precipitating solutions have less layer-averaged liquid water (Fig. 1), the greater updraft-downdraft asymmetry is responsible for a greater loading effect. Both simulations receive similar contributions to the buoyancy production of TKE from vapor fluctuations. Overall, the buoyancy production of TKE is trapped in a shallower layer in experiment HDB; downdrafts lose their buoyancy and contribute to the midlevel peak in $\overline{u^{\prime} u^{\prime}}+\overline{v^{\prime} v^{\prime}}$ near cloud base (Fig. 12).

\section{Potential buoyancy and subcloud-layer cooling}

The above discussion illustrates how, by stabilizing downdrafts, precipitation imposes an asymmetry on the circulation. But the question remains: why are downdrafts stabilized in the presence of precipitation? The net removal of liquid water from a parcel immediately effects neither $\theta$ nor $r_{v}$ and thus only directly affects the buoyancy production of TKE through a reduction in the liquid-water loading term. Hence, in the upper portion of the cloud, the buoyancy of parcels may be increased locally; this might explain the kink (beginning at $z=0.85 z_{i}$ through $z=0.9 z_{i}$ ) in the updraft-averaged buoyancy production in Fig. 11b, but it does not explain the effect of drizzle on the buoyancy production of TKE by downdrafts.

A closer analysis indicates that drizzle affects the dynamics of the circulation through two distinct, but related, processes. These processes are delineated in Fig. 13, a plot of the diabatic terms that shows a narrow region of intense drizzle flux divergence near cloud top (which we associate with an effective heating on the same order as the radiative cooling) overlays a deeper region of flux convergence (associated with cooling). The first, for reasons which will become clear, we call "potential buoyancy." It is associated with the implied "heating" or flux divergence at cloud top. The second, is associated with the evaporation of drizzle below cloud base. The processes are distinct but related in that the first process need not imply the second (all the drizzle could conceivably fall to the ground with very little or no subcloud evaporation) but the second does imply the first. Each process is discussed in turn below

Generically speaking, it is the divergence in the radiative flux at cloud top that cools parcels, thus reducing their buoyancy and destabilizing the cloud layer. In response to radiative cooling, negatively buoyant air collects and forms downdrafts, which are the manifestation of the TKE and the agents of PBL mixing. If downdrafts 

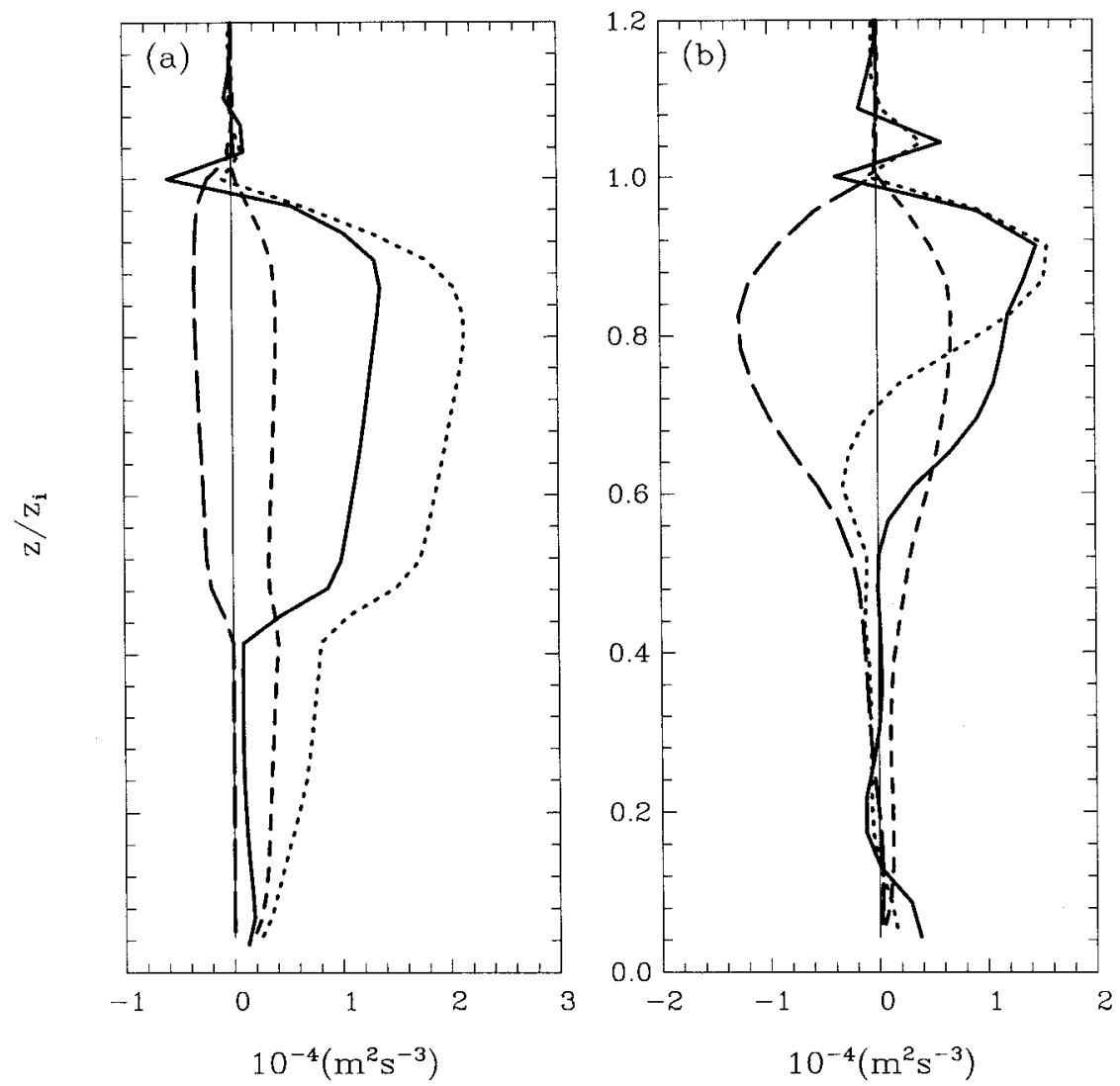

FIG. 11. Partition of buoyancy flux: (a) experiment NDB and (b) experiment HDB. Contribution by updrafts (solid), contribution by downdrafts (dotted), contribution by vapor fluxes (short-dash), and liquid water loading (long-dash). Note that the up- and downdraft contributions sum to the total buoyancy flux as plotted in Fig. 9.

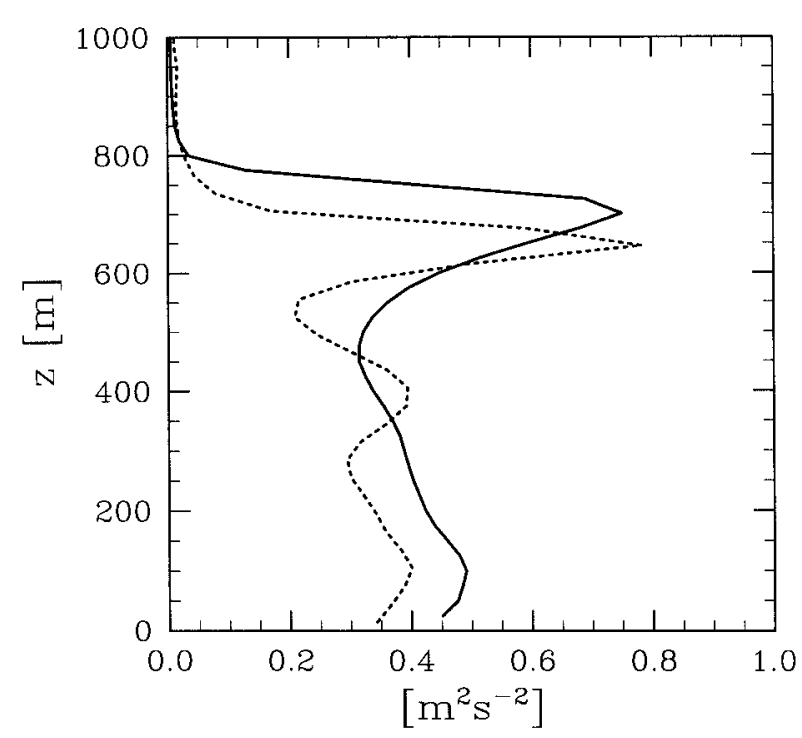

FIG. 12. Variances in horizontal velocities averaged over third hour for simulations HDB (dotted) and NDB (solid).

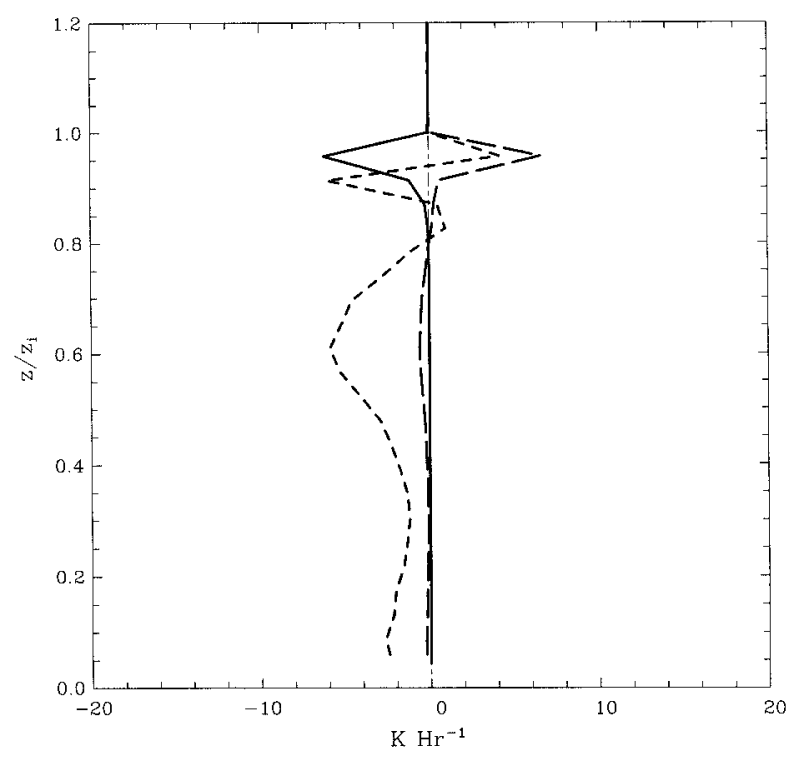

FIG. 13. The $\theta_{l}$ source terms for simulation HDB averaged over third hour: $\partial F_{\text {rad }} / \partial z$ (solid), $-\partial F_{\mathrm{drz}} / \partial z$ (long dash), and $10\left(\partial F_{\mathrm{rad}} / \partial z-\right.$ $\left.\partial F_{\mathrm{drz}} / \partial z\right)$ (short dashed). 


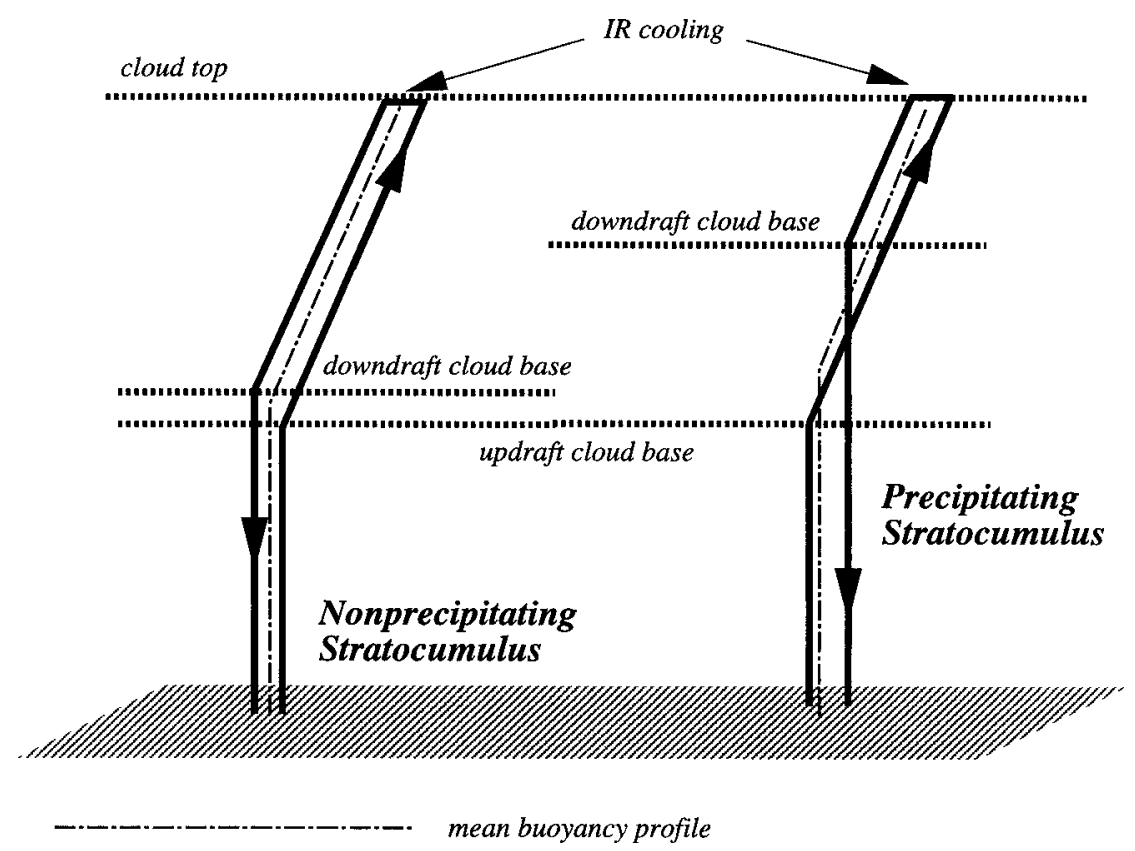

FIG. 14. Conceptual cartoon of hypothetical parcel trajectories in $\left(\theta_{v}, z\right)$ space for precipitating and nonprecipitating PBLs.

and updrafts have similar saturation levels, buoyancyflux jumps across cloud base are lessened and radiative cooling can lead to a deep layer of buoyancy generation of TKE (Schubert et al. 1979; Bretherton and Wyant 1997). This favors the formation of deep circulations, which helps maintain a well-mixed layer. If precipitation formation is active, parcels lose substantial amounts of liquid water (cf. Figs. 3 and 4), which imposes an asymmetry on their ascending and descending saturation heights. As a result, the saturation level for downdrafts will be above that for updrafts and descending parcels follow a moist adiabat over a shorter distance relative to ascending parcels. This process tends to stabilize downdrafts with respect to deeper mixing.

In a manner peculiar to fluids that do not conserve buoyancy, the net condensational heating associated with precipitation formation is in effect a potential buoyancy: while it is imparted near cloud top, it is only realized once the downdraft parcel becomes subsaturated. These processes are schematically represented by the cartoon in Fig. 14. Here, the hypothetical trajectory of a parcel that mixes through the whole boundary layer is shown. ${ }^{3}$ In the nonprecipitating PBL, the parcel generates TKE at all times; it is buoyant when going up and negatively buoyant when going down. In the precipitating solutions, the updrafts tend to be negatively buoyant in the subcloud layer but positively buoyant

\footnotetext{
${ }^{3}$ By hypothetical it is meant that this figure denotes the buoyancy a parcel would have were it to complete a circuit. In all likelihood, parcels do not circulate in this manner in the presence of precipitation.
}

above. The cloud layer is doing work on the subcloud layer, something actually observed in the simulations. Because the radiative cooling at cloud top is not sufficient to offset the effects of net condensation, parcels moving downward rapidly become buoyant and have insufficient kinetic energy to complete the circuit.

For the reasons discussed above, in the precipitating case there is insufficient turbulent energy creation to allow downdraft parcels to mix to the surface. As discussed theoretically (e.g., Schubert et al. 1979; Bretherton and Wyant 1997) entrainment has a similar effect on the dynamics, a fact well supported by detailed calculations (e.g., Krueger et al. 1995b). However, there is an important difference between entrainment-induced potential buoyancy and precipitation-induced potential buoyancy, as for a given subsidence velocity the potential buoyancy imparted by precipitation falling from a parcel does not require the boundary layer to deepen as rapidly; indeed, it tends to inhibit such deepening. Another difference between the two types of potential buoyancy is that entraining downdraft parcels often see an immediate impact on their buoyancy; as drier mixedin air is often warmer, precipitating parcels only see the effect of the net-condensational heating of the parcel at cloud base. To summarize, in the precipitating simulations, radiative cooling can promote the formation of negatively buoyant parcels in a shallow layer, but precipitation stabilizes these parcels with respect to deeper mixing. This is consistent with both the differences in the circulations evidenced in the snapshots (cf. Figs. 3b and $4 \mathrm{~b}$ ), and the updraft-downdraft partitioned buoyancy flux term in Fig. 11. 
The second manner in which precipitation interacts with the dynamical evolution of the boundary layer is through its cooling and moistening of the subcloud layer. For the case of light drizzle, Wang and Wang (1994) suggest that this process is responsible for the reduction of TKE production in the PBL. However, their model is one-dimensional, and despite assumptions about the distribution of liquid water, it is not well suited to a study of the effects of drizzle on up- and downdraft asymmetries. The effect of a stabilized cloud layer on the turbulent circulations is important to the dynamics illustrated by the LES; drizzle generates about $1 \mathrm{~K}$ of stabilization in the subcloud layer (see Fig. 1), which effectively reduces the potential of downdrafts to mix a deep layer. The tendency of drizzle to stratify the PBL, thereby constructing a potential barrier to deep mixing by downdrafts, most likely cooperates with the potential buoyancy imparted by precipitation formation and enhances the drizzle-induced stratification of the subcloud layer relative to the cloud layer. The inability of circulations forced at cloud top to maintain a well-mixed layer allows conditional instability to develop, thereby setting the stage for more cumulus-like dynamics. In addition to providing an alternate source of TKE production in the precipitating calculations, the generation of cumulus updrafts is also important in maintaining the coupling between the subcloud and cloud layers, this in turn provides the moisture flux necessary to sustain large drizzle rates.

Ideally, we would like to understand how much of the dynamics associated with precipitating PBLs can be explained solely in terms of subcloud evaporative effects (subcloud layer cooling) versus cloud layer heating (potential buoyancy) effects. Unfortunately, the realism of our microphysical parameterization (compared to most bulk microphysical schemes that diagnose cloud water) makes such a study difficult because a qualitative distinction between drizzle and cloud drops is not made in our model, in that drizzle drops cannot be prevented from evaporating without preventing cloud drops from evaporating. This remains an open issue that the authors plan to examine further using a simpler microphysical model. Our current results, produced using a detailed microphysical model, should prove to be a useful benchmark for such a study.

\section{Discussion}

Here we return to some of the questions raised in the introduction. Specifically we recall how various hypotheses relating changes to $\mathrm{CCN}$ concentrations (mediated by their effect on the efficiency with which PBL clouds produce precipitation) to larger-scale effects depended on simplified assumptions about the nature of precipitating PBLs. While it is difficult to definitively answer general questions about the role of drizzle on the PBL based on a couple of 3-h case studies, we can see if the basic dynamics of our simulations are consistent with various scenarios.

\section{a. Decoupling and PBL evolution}

A persistent question about the effect of drizzle on the PBL is whether or not it induces decoupling. The answer to this question, which has broad implications for a variety of conceptual models of drizzle, depends in part upon one's definition of decoupling. "Decoupling" is a carelessly used term in the meteorological literature. Some just associate decoupling with stratification and fail to appreciate that a PBL can be poorly mixed but strongly coupled. Original explorations of the concept (Nicholls 1984) focused on diurnal decoupling and were more rigorous in their definition: decoupled PBLs were modeled as ones in which all fluxes vanished at some point, or buoyancy fluxes vanished over some spatial interval (Turton and Nicholls 1987). If one adopts this more stringent definition, then drizzle does not induce decoupling in our simulations because fluxes of $\theta_{l}$ and $q_{t}$ do not vanish. They do not even have local minima. Heavy drizzle does, however, force a marked change in the nature of the PBL coupling. Simulation HDB suggests that, for buoyancy-driven flows, turbulent fluxes of conserved quantities may be reduced in the subcloud layer (see, for instance, the turbulent flux of total water below $z=0.3 z_{i}$ ), which in turn may cause the PBL to evolve toward a state of intermittent cloudiness. However, when enough shear is present, quasisteady precipitating solutions, in which turbulent fluxes of conserved quantities are everywhere increased, appear possible. In these simulations the presence of stratification inhibits regular radiatively driven convection typical of stratocumulus; instead, cumulus clouds are the primary agents through which the cloud and subcloud layer are coupled. That is not to say that shear, or a strong mean wind, is necessary for the effective coupling of the cloud and subcloud layer when precipitation is heavy. Irrespective of the strength of the mean wind, drizzle generates conditional instability in the subcloud layer and cumulus clouds form. The cumulus convection is simply more effective at coupling the cloud and subcloud layer when a strong mean wind helps ventilate the surface and promote conditional instability.

It remains unclear to what extent the dynamics discussed above are an artifact of our initial conditions, which had a neutrally stratified surface layer. For this choice of initial conditions the subcloud evaporation of drizzle can efficiently modulate surface sensible heat fluxes thereby helping to initiate cumulus convection. If stratocumulus-topped PBLs begin drizzling over much colder water, cumulus coupling may never occur, in which case drizzle may effectively deplete cloud LWP, thereby reducing turbulent energy production, mixing, and cloud lifetimes. However, such a scenario, while consistent with rigorous measures of decoupling and the conceptual model of Paluch and Lenschow 

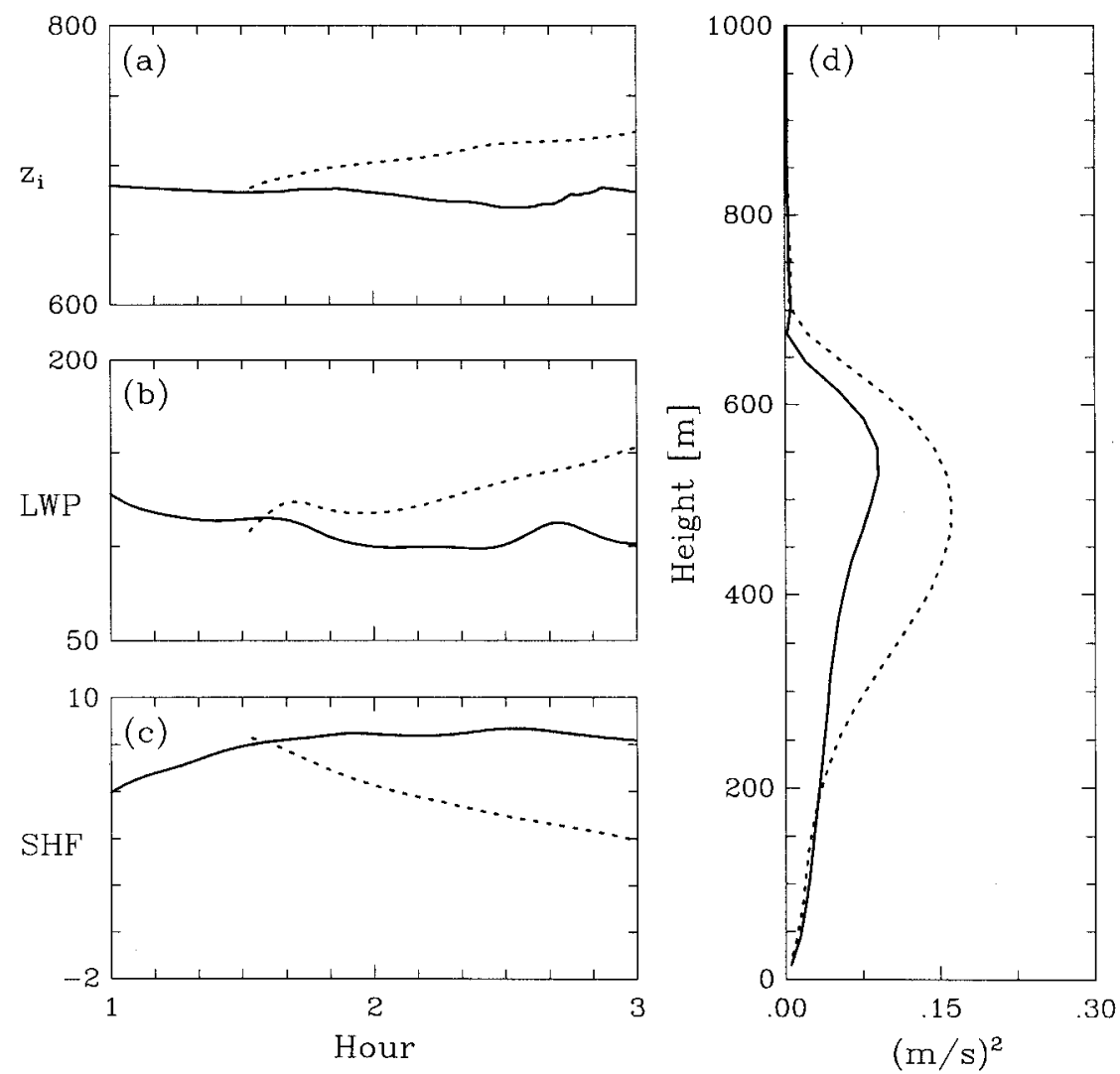

FIG. 15. HDBS (solid line) and HDBS sensitivity study (drizzle turned off after hour 1.5): (a) inversion height, (b) vertically integrated liquid water, (c) sensible-heat flux, and (d) $\overline{w^{\prime} w^{\prime}}$ averaged over third hour.

(1991), would not be characteristic of the downstream evolution of subtropical stratocumulus decks, which are known to move over warmer water.

\section{b. The stratocumulus to trade cumulus transition}

As previously discussed, the dynamics of our precipitating simulations are similar to simulations of the equatorward downstream evolution of stratocumulus in the absence of precipitation as illustrated by two-dimensional models of varying complexity (Krueger et al. 1995a,b; Wyant et al. 1997; Bretherton and Wyant 1997). To review, these two-dimensional simulations suggest that as stratocumulus-topped PBLs move over warmer water, they deepen and are unable to maintain a well-mixed state. More cumulus-like dynamics result as the shallow stratocumulus-topped PBLs gradually evolve into a more trade-cumulus-like regime. An important difference between these dynamics and those illustrated by our precipitating solutions is that PBL growth is reduced when precipitation is active. Hence, precipitation does not appear to be inducing an irreversible change in PBL structure. Here we have equated PBL deepening with an irreversible change because the timescales of reestablishing a sharp shallow inversion (given a deep, trade-wind-like, boundary layer in which SSTs are reset to their initial, lower values) are long compared to the timescales of interest (i.e., a few hours). The concept of reversibility in the precipitating solutions reflects our idea that upon the cessation of precipitation a shallow well-mixed boundary layer will be reestablished on these shorter timescales.

To test this idea of reversibility, we examined the effect of artificially stopping drizzle in integration HDBS after $90 \mathrm{~min}$. In the subsequent $90 \mathrm{~min}$, the PBL rapidly evolved back toward a well-mixed state and the dynamics characteristic of such a state. As illustrated by Fig. 15, $\overline{w^{\prime} w^{\prime}}$ averaged over the third hour is significantly larger once drizzle has been turned off. LWP increases steadily with time, and the mixing-out of the surface layer (i.e., the reestablishment of a mixed layer in which internal gradients of conserved variables vanish) is reflected in the steadily decreasing surface sensible heat fluxes. That the entrainment rate almost immediately approaches values characteristic of the nonprecipitating solutions indicates that the potential buoyancy effect of drizzle is the primary process responsible for reduced entrainment rates. If this tendency toward recovery is a robust feature of heavily drizzling PBLs, the efficient production of precipitation may (if the 
break in cloud fraction is associated with boundary layer deepening) lead to larger time-averaged cloud fractions but smaller instantaneous LWPs for completely cloudy states. The net effect of such dynamics on the radiative balance is not obvious.

\section{c. The role of cloud depth on TKE production}

As noted in the introduction, previous studies have shown that drizzle leads to reduced amounts of boundary layer TKE. However, explanations as to the reason for this have varied. Because our initial sounding is particularly moist above the inversion, clouds deepen as the PBL grows. Observations suggest that this type of sounding is common, as is the simulated response of the cloud (Austin et al. 1995; Brost et al. 1982a). When entrainment leads to cloud deepening and if the net buoyancy flux scales with the depth of the cloud layer, then entrainment (which is thought to scale with the net buoyancy flux) should decrease as the cloud layer thins. Consequently, when comparing simulations, values of $\overline{w^{\prime} w^{\prime}}$ should be positively correlated with both entrainment and cloud LWP. Does that mean that one effect causes the others and, if so, is one effect primary? As discussed in the introduction, previous investigators have suggested that less liquid water results when drizzle is effective at removing water from the cloud; the resulting thinner cloud then experiences smaller cloudtop radiative cooling rates, smaller values of TKE, and less entrainment.

Does this set of physical processes explain what is happening in our simulations? Because we use a simple radiative parameterization, this question is readily answered by numerical experiment. Recollect that the radiative cooling is proportional to the divergence of the radiative flux,

$$
\left.c_{p} \rho_{0} \frac{d \theta}{d t}\right|_{\mathrm{rad}}=\frac{\partial F_{\mathrm{rad}}}{\partial z}
$$

and that a thicker cloud (which has more liquid water at cloud top) experiences a sharper change in the radiative flux, thereby implying larger cloud-top radiative cooling rates, even if the total change in the radiative flux (and the mass-weighted cooling rate) across the entire cloud is unaffected. To see if the changes in the depth of the cooling layer were important, simulation NDB was repeated but with a smaller absorption coefficient. We set $\alpha=65$ in Eq. (6), which is half its value in the control. This mimics the effect on the mean radiation budget of a cloud with layer-averaged LWPs reduced by a factor of 2 ; it is also consistent with the differences between the precipitating and nonprecipitating integrations (cf Fig. 1c). We found that halving the depth of the cooling layer had no significant influence on the statistics of the integration. This suggests that the radiative consequence of drizzle-induced changes to the cloud layer plays a relatively minor role in the dynamical response of the simulation to drizzle.

\section{d. Boundary layer collapse?}

What about the idea that precipitating PBLs might collapse? ${ }^{4}$ Our simulations support the findings of Ackerman et al. (1993), especially when shear production of TKE is small; although, as previously noted above we offer a different explanation for the dynamics behind such a collapse. Our results also suggest that shear production of TKE promotes larger precipitation rates but that larger precipitation rates need not imply more rapid collapse relative to the purely buoyancy driven case. This differs from what was found in a sensitivity study performed by Ackerman et al., as they showed that larger precipitation rates in a more shear-driven PBL promotes a more rapid collapse. We believe that the difference between our result and theirs may be an artifact of their approach, as a one-dimensional model has difficulty in properly representing the more cumulus-like dynamics of precipitating PBLs [see the discussion of Wang and Wang (1994) on this point]. Our results further suggest that an accurate representation of the cumulus coupling of heavily drizzling PBLs is essential in representing their subsequent evolution. This means that such processes must be properly accounted for in higher-order closure models and that PBL models must have at least two layers.

\section{e. What sets the drizzle rate?}

Nicholls (1987) suggested that an important role of drizzle may be to limit cloud LWP. If this is true, and drizzle merely limits the vertically integrated cloud water, then it might be quite easy to represent parametrically. For instance, one parameterization could be that the cloud-base drizzle rate is whatever it takes to prevent the LWP from exceeding a certain threshold, where this threshold is a function of the efficiency with which the cloud produces precipitation.

Our simulations provide some initial support for such a view. Drizzle rates are approximately $50 \%$ greater in experiment HDBS relative to experiment HDB (which has much lighter mean winds); however, the LWP in each simulation is nearly equal (cf. Figs. 1 and 2). Because of the design of the experiments (similar external forcing and background $\mathrm{CCN}$ distribution) we would not expect the precipitation efficiency of the two calculations to be substantially different, thus our results do not contradict the idea that for a given subcloud CCN distribution and a given level of buoyancy production

\footnotetext{
${ }^{4}$ Collapse is a term coined by Ackerman et al. (1993); although it has a connotation of suddenness, it is merely used to describe the shallowing of a boundary layer in which large-scale subsidence is much greater than the mean entrainment rate.
} 
within the cloud layer there exists a single equilibrium liquid water path. One limiting factor to consider might be the relationship between the maximum radiative forcing and the maximum drizzle flux. If the maximum value of $\bar{F}_{\mathrm{drz}}$ significantly exceeds the change in $\bar{F}_{\text {rad }}$ across the cloud layer, it would seem difficult to maintain a quasi-steady stratocumulus-topped PBL.

\section{A testable hypothesis?}

Hypothesis: Persistent, well-mixed, shallow, radiatively driven stratocumulus-topped PBLs, in which drizzle is heavy and downdrafts are negatively buoyant through a deep layer, do not exist in nature.

Because such boundary layers are readily observed in the absence of persistent drizzle, we believe that our hypothesis, despite its many qualifications, is readily testable. The qualifications, however, warrant further clarification. While the effects of drizzle that we discuss may be more broadly applicable, most of the qualifications reflect that only a limited regime in parameter space has been explored, that is, shallow, heavily precipitating, stratocumulus-topped PBLs in which the surface air is in near-thermal balance with the underlying water. By shallow, we mean boundary layers with $z_{i}<$ $1000 \mathrm{~m}$ (700 $\mathrm{m}$ in our case); it may well be that drizzle in deeper layers (for example $1500 \mathrm{~m}$ deep) affects the dynamics quite differently. And as discussed above, drizzle over cold water may well result in more readily separable cloud and subcloud layers. Heavily precipitating PBLs refers to those in which the maximum drizzle flux is commensurate with the radiative forcing. Our simulations were also based on the idealization of an invariant $\mathrm{CCN}$ distribution. But because the dynamics adjust to the precipitation on a timescale of an eddy turnover time, as long as this is small compared to an $e$-folding time in the $\mathrm{CCN}$ concentrations, we might expect the steady-state $\mathrm{CCN}$ distribution experiments to have physical relevance. Notwithstanding these qualifications, the dynamics illustrated by the simulations, if realistic, appear to be robust enough to be observable.

To elaborate on our hypothesis recall that the precipitating calculations are considerably more inhomogeneous. Cumulus clouds are forming out of a moistened subcloud layer; furthermore, they appear to be associated with larger-scale circulations as well as more spatial variability in the LWP. ${ }^{5}$ Our experiences with LES suggest that such a situation, while clearly evident in shallow $\left(z_{i}<1000 \mathrm{~m}\right)$, heavily precipitating PBLs, is rare in a shallow, nonprecipitating PBL, particularly in the presence of sufficient radiative forcing. There is casual

\footnotetext{
${ }^{5}$ If variability in low-cloud LWP and larger-scale organization could be robustly related to drizzle, this in conjunction with standard remote sensing techniques might provide a valuable means for better assessing the frequency of heavy drizzle in cloud-topped PBLs.
}

observational support for this view as cumulus clouds are often reported in PBLs with strong drizzle (e.g., Bretherton et al. 1995; Martin et al. 1995). In addition, significant signatures of the precipitating solutions are the substantial stratification across the mean cloud-base level, reduced values of turbulent kinetic energy, and a cloud-base flattening of downdrafts. The reduction in $\overline{w^{\prime} w^{\prime}}$ is dramatic, particularly in the subcloud layer and should be observable. Although locally, convective eddies could be as strong as in nondrizzling integrations, these eddies tended to be intermittent in space and time and inevitably associated with cumulus convection. The absence of regularly spaced, deeply penetrating downdrafts also distinguished the precipitating integrations from the nonprecipitating ones. The cloud-base peak in $\overline{u^{\prime} u^{\prime}+v^{\prime} v^{\prime}}$ (see Fig. 12) might also be observable, perhaps by a cloud radar operated at shallow scan angles.

In summary, for various reasons, one or the other of the above delineated features may be observed in nonprecipitating PBLs. We are claiming them all to be collectively characteristic of heavily precipitating, shallow, stratocumulus-topped PBLs. In other words, if there ever were some observations of persistent, well-mixed, shallow, radiatively driven stratocumulus-topped PBLs in which drizzle is heavy and downdrafts are penetrative (i.e., they are negatively buoyant through a deep layer), it would force us to reexamine the relationship between our model and reality. Despite the number of qualifications, shallow, well-mixed, radiatively driven stratocumulus-topped PBLs in which drizzle is heavy and downdrafts are penetrative are common in nature in the absence of drizzle. Thus our hypothesis, that they do not exist in the presence of strong drizzle, seems to stand a reasonable chance of failing, and thus is testable.

\section{Summary}

LESs of heavily and nonprecipitating stratocumulustopped PBLs in two PBL regimes have been presented. One regime is characterized by buoyancy production of TKE; the other has nearly equal contributions to TKE production from shear and buoyancy. The precipitating simulations are characterized by reduced buoyancy fluxes and smaller values of $\overline{w^{\prime} w^{\prime}}$, a moistened and cooled subcloud layer, less entrainment, reduced LWP, but more horizontal variability. As has been previously suggested (Nicholls 1987), drizzle appears to limit cloud LWP. In contrast to nonprecipitating stratocumulus layers, cumuli forming out of the subcloud layer contribute significantly to the dynamics of heavily drizzling PBLs. These cumuli are effective at coupling the cloud and subcloud layers, particularly when larger wind speeds help ventilate the surface, thereby generating conditional instability and sustaining TKE in the subcloud layer. A sensitivity study further indicates that in contrast to cumulus-coupled PBLs associated with an increase in SSTs, the precipitating solutions have the char- 
acter of a forced solution, as upon the cessation of precipitation the PBL recovers its nonprecipitating state (and dynamics) on a timescale of a few hours.

Previous studies (Chen and Cotton 1987; Ackerman et al. 1993) have suggested that drizzle impacts the dynamics primarily by changing the radiative properties of the clouds. Our sensitivity study, in which the optical properties of nonprecipitating clouds were modified to mimic the changes in optical properties associated with heavy drizzle, indicates that this is not the case in our simulations.

Wang and Wang (1994) argue that the predominant effect of light drizzle is to stratify the subcloud layer, thereby reducing the buoyancy production in the mean cloud layer. In our simulations, drizzle generates potential buoyancy in parcels near cloud top. By this we mean that buoyancy, or heating, is imparted via a reduction in the saturation levels, thus stabilizing them against deeper mixing. This primarily reduces buoyancy production in the cloud layer and probably cooperates with the tendency of drizzle to evaporate in and stabilize the subcloud layer. The net result of such cooperation is a cooled and moistened subcloud layer that promotes enhanced surface sensible heat fluxes and is more prone to cumulus convection.

In our simulations, in which SSTs were initialized in near-thermal equilibrium with the air above, drizzle did not lead to a decoupling of the PBL. Instead, the nature of the coupling changed-it became more cumulus like. This cumulus coupling was more efficient in transporting heat and moisture; turbulent fluxes of $\theta_{l}$ and $q_{t}$ were actually enhanced despite the reduction in vertical velocity variances. The suggestion that drizzle induces decoupling might hold for the case of stratocumulus layers over much colder water. However, in contrast to earlier hypotheses (Paluch and Lenschow 1991), our results suggest that drizzle-induced decoupling (i.e., the cessation of transport of moisture out of the subcloud layer) probably is not characteristic of the downstream evolution of stratocumulus moving over warmer water.

Lastly, the tendency of drizzle to reduce TKE production and entrainment suggests that cloud layers may remain shallower for longer periods of time. Hence, while heavy drizzle may reduce cloud-averaged optical depths to the extent that cloud fractions are correlated with PBL depth, drizzle may increase time-averaged cloud fractions, thereby making the influence of drizzle on the radiation budget ambiguous. Moreover, light drizzle-by reducing entrainment in PBLs with large jumps in moisture across the inversion-might actually lessen entrainment drying thereby leading to deeper PBL clouds. Such scenarios are largely speculative and need to be considered further.

Acknowledgments. This work stems from BS's doctoral research at CSU. During this time, he gratefully acknowledges funding from a NASA Graduate Fellowship on Global Change, Grant NGT-30231. Additional support for GF and WRC was provided by NSF Grant ATM-9529321. The comments of J. Wilczak, H. Gerber, $\mathrm{S}$. Krueger, and one anonymous reviewer greatly improved the clarity of this manuscript. BS would also like to acknowledge fruitful interactions with the GCSS Working Group One members; conversations with Don Lenschow and Ilga Paluch also proved stimulating. The last stages of this work benefitted gratefully from BS's sponsorship by the NCAR's Advanced Study Program. $\mathrm{Al}$ Cooper is thanked for making it such a pleasant place to spend one's time.

\section{APPENDIX \\ Model Changes}

\section{a. Subgrid model}

The subgrid model has been modified slightly from what was described in previous studies; presently eddy diffusivities and viscosities are solved for at $w$ points. Also the filter scale $l$ is weighted to account for the distance $z$ between a grid level and the surface:

$$
l^{2}=\frac{\left(c_{s} \Delta x\right)^{2}}{1+\left(\frac{c_{s} \Delta x}{k z}\right)} .
$$

Here $k$ is von Kármán's constant, and $c_{s}=0.22$ is a dimensionless constant derived from inertial-range arguments (Lilly 1967; Mason 1994).

\section{b. Infrared radiation}

Because our primary interest is in how drizzle interacts with the turbulence, we now use a very simple parameterization of the cloud-top radiative forcing. This parameterization is based on a graybody approximation in which radiative fluxes respond only to condensate mixing ratio, $q_{l}$ :

$$
F_{\mathrm{rad}}(z)=F_{0} e^{-\alpha(\mathrm{LWP}(z))},
$$

where

$$
\operatorname{LWP}(z)=\int_{z}^{z_{\text {top }}} \rho_{0} q_{l} d z,
$$

$F_{0}$ is the maximum rate at which energy that can be extracted from a unit area of the flow, $\alpha$ is a parameter that regulates the depth of the cloud layer over which this extraction takes place, $\rho_{0}$ is the basic-state density, $z_{\text {top }}$ is the model top, and the integral is approximated by a midpoint Riemann sum. In the present study we specify $\alpha=130 \mathrm{~m}^{2} \mathrm{~kg}^{-1}$ and $F_{0}=74 \mathrm{~W} \mathrm{~m}^{-2}$.

\section{c. Ventilative enhancement of droplet evaporation}

Originally, we used the analytically integrable form,

$$
\frac{d m}{d t}=C(p, T) \eta(t) \frac{m^{2 / 3}}{m^{1 / 3}+l_{0}},
$$


as the basis for describing how drops grow or shrink due to condensation or evaporation. Here $C(p, T)$ is a thermodynamic coefficient dependent on pressure $p$ and temperature $T ; m$ is the mass of a drop and $l_{0}$ is a length scale introduced to model gas-kinetic effects. The timedependent function $\eta(t)$ is the difference between the saturation mixing ratio and the water-vapor mixing ratio; when it is positive, the drop mass increases due to condensation, and when negative, drops evaporate. When drops move relative to the airstream, fluxes of heat and vapor are more efficient, and the following equation better describes their evolution in time:

$$
\frac{d m}{d t}=C(p, T) \eta(t) \frac{m^{2 / 3}}{m^{1 / 3}+l_{0}} \overline{f_{v}(m)},
$$

where $f_{v}(m)$ is an empirically determined factor that multiplies the growth equation. Following Pruppacher and Klett (1978), we write

$$
\overline{f_{v}(m)}= \begin{cases}1.00+0.1\left(N_{\mathrm{Sc}}^{1 / 3} N_{\mathrm{Re}}^{1 / 2}\right)^{2}, & N_{\mathrm{Re}} \leq 2 \\ 0.75+0.3\left(N_{\mathrm{Sc}}^{1 / 3} N_{\mathrm{Re}}^{1 / 2}\right), & N_{\mathrm{Re}}>2,\end{cases}
$$

where $N_{\mathrm{Sc}}$ is the Schmidt number (the ratio of kinematic viscosity to diffusivity) and $N_{\mathrm{Re}}=u_{\infty} d / \nu$ is the Reynolds number; here $u_{\infty}$ is the drop terminal velocity, $\nu$ is viscosity, and $d$ is drop diameter. Because $u_{\infty}$ is a function only of mass, $\overline{f_{v}}$ only has a mass dependence.

In our microphysical parameterization, drops are organized into 25 size intervals (bins) with mass doubling between bins. The smallest mass represented is $16 \times$ $10^{-12} \mathrm{~g}$, and the largest is about $0.5 \times 10^{-3} \mathrm{~g} ; \overline{f_{v}}$ does not become significantly larger than unity until drops reach bin 15 (approximately $262 \times 10^{-9} \mathrm{~g}$ ). Drops rarely reach the upper size limit for which $\overline{f_{v}} \approx 6$. Clearly in heavily precipitating situations, the inclusion of ventilative effects may be important. For $N_{\mathrm{Re}} \leq 2$, drop terminal velocities are well described by Stokes flow so that $N_{\operatorname{Re}} \propto m$. Drops larger than this size but smaller than the largest drop represented by the model are well described by $N_{\mathrm{Re}} \propto m^{2 / 3}$, which implies that

$$
\overline{f_{v}(m)}= \begin{cases}c_{1}+c_{2} m & N_{\mathrm{Re}} \leq 2 \\ c_{3}+c_{4} m^{1 / 3} & N_{\mathrm{Re}}>2,\end{cases}
$$

with $c_{i}$ being a positive constant. Unfortunately, solutions of Eq. (A4) for $m(t)$ using the above forms for $\overline{f_{v}(m)}$ are implicit and must be iteratively solved. Alternatively, Eq. (A4) may be numerically integrated or approximated such that its integrals are simple analytic expressions.

The nature of our semi-Lagrangian condensation/ evaporation scheme (Stevens et al. 1996) requires us to find how large or small drops will grow or evaporate under a given forcing. Each bin is solved for individually. This means that Eq. (A4) must only be solved locally. Consequently, linearizing the integrand, or assuming mass to be constant in part of the expression, may generate approximate expressions for $m(t+\Delta t)$, whose accuracy is reasonably good. After some experimentation, it was found that for our purposes Eq. (A4) is well approximated by assuming $\overline{f_{v}}(m)=\overline{f_{v}}\left(m_{k}\right)$ for $m \in\left[m_{k}, m_{k+1}\right]$. Thus the analytic form of our solution to the drop growth equation without ventilation effects must only be multiplied by a constant factor that depends on the drop bin in which the drop originally resides.

In other words, sufficiently good accuracy is obtained by neglecting the change in the ventilation effect experienced by a drop during a single time step. Such an approximation is consistent with the calculation of the mean supersaturation over the time step, where it is assumed that the integral radius of the droplet spectrum is given by its value at the beginning of the time step.

\section{REFERENCES}

Ackerman, A. S., O. B. Toon, and P. V. Hobbs, 1993: Dissipation of marine stratiform clouds and collapse of the marine boundary layer due to the depletion of cloud condensation nuclei by clouds. Science, 262, 226-229.

Albrecht, B. A., 1989: Aerosols, cloud microphysics and fractional cloudiness. Science, 245, 1227-1230.

Austin, P., Y. Wang, R. Pincus, and V. Kujala, 1995: Precipitation in stratocumulus clouds: Observational and modeling results. $J$. Atmos. Sci., 52, 2329-2352.

Bjerknes, J., 1938: Saturated-adiabatic ascent of air through dryadiabatically descending environment. Quart. J. Roy. Meteor. Soc., 64, 325-330.

Boers, R., J. B. Jensen, P. B. Krummel, and H. Gerber, 1996: Microphysical and short-wave radiative structure of wintertime stratocumulus clouds over the southern ocean. Quart. J. Roy. Meteor. Soc., 122, 1307-1340.

Bretherton, C. S., and M. C. Wyant, 1997: Moisture transport, lowertropospheric stability, and decoupling of cloud-topped boundary layers. J. Atmos. Sci., 54, 148-167.

- P. Austin, and S. T. Siems, 1995: Cloudiness and marine boundary layer dynamics in the ASTEX Lagrangian experiments. Part II: Cloudiness, drizzle, surface fluxes, and entrainment. J. Atmos. Sci., 52, 2724-2735.

Brost, R. A., D. H. Lenschow, and J. C. Wyngaard, 1982a: Marine stratocumulus layers. Part I: Mean conditions. J. Atmos. Sci., 39, 800-817.

- J. C. Wyngaard, and D. H. Lenschow, 1982b: Marine stratocumulus layers. Part II: Turbulence budgets. J. Atmos. Sci., 39, $818-836$.

Chen, C., and W. R. Cotton, 1987: The physics of the marine stratocumulus-capped mixed layer. J. Atmos. Sci., 44, 2951-2977.

De Roode, S. R., and P. G. Duynkerke, 1996: Dynamics of cumulus rising into stratocumuls as observed during the first "Lagrangian" experiment of ASTEX. Quart. J. Roy. Meteor. Soc., 122, $1597-1624$

Feingold, G., B. Stevens, W. R. Cotton, and A. S. Frisch, 1996: The relationship between drop in-cloud residence time and drizzle production in numerically simulated stratocumulus clouds. $J$. Atmos. Sci., 53, 1108-1122.

, R. L. Walko, B. Stevens, and W. R. Cotton, 1998: Simulations of marine stratocumulus using a new microphysical parameterization scheme. J. Atmos. Res., in press.

Gerber, H., 1996: Microphysics of marine stratocumulus clouds with two drizzle models. J. Atmos. Sci., 53, 1649-1662.

Gunn, R., and G. D. Kinzer, 1949: The terminal velocity of fall for water drops in stagnant air. J. Meteor., 6, 243-248.

Hall, W. D., 1980: A detailed microphysical model within a two- 
dimensional dynamical framework: Model description and preliminary results. J. Atmos. Sci., 37, 2486-2507.

Jones, R. F., 1951: Rain from non freezing clouds. Meteor. Mag., 80, 273-274

Kogan, Y. L., M. P. Khairoutdinov, D. K. Lilly, Z. N. Kogan, and Q. Liu, 1995: Modeling of stratocumulus cloud layers in a large eddy simulation model with explicit microphysics. J. Atmos. Sci., 52, 2293-2940.

Kraus, E. B., 1963: The diurnal precipitation change over the sea. $J$. Atmos. Sci., 20, 551-556.

Krueger, S. K., G. T. McLean, and Q. Fu, 1995a: Numerical simulation of stratus-to-cumulus transition in the subtropical marine boundary layer. Part I: Boundary layer structure. J. Atmos. Sci., 52, $2839-2850$

$[,-$, and $-1995 \mathrm{~b}$ : Numerical simulation of stratus-tocumulus transition in the subtropical marine boundary layer. Part II: Boundary layer circulation. J. Atmos. Sci., 52, 2851-2868.

Lilly, D., 1967: The representation of small-scale turbulence in numerical simulation experiments. Proceedings of the IBM Scientific Computing Symposium on Environmental Sciences, H. H. Goldstine, Ed., IBM Publ. 320-1951, 195-210.

Long, A. B., 1974: Solutions to the droplet coalescence equation for polynomial kernels. J. Atmos. Sci., 31, 1040-1052.

Martin, G. M., D. W. Johnson, D. P. Rogers, P. R. Jonas, P. Minnis, and D. A. Hegg, 1995: Observations of the interaction between cumulus clouds and warm stratocumulus clouds in the marine boundary layer during ASTEX. J. Atmos. Sci., 52, 2902-2922.

Mason, B. J., and B. P. Howarth, 1952: Some characteristics of stratiform clouds over North Ireland in relation to their precipitation. Quart. J. Roy. Meteor. Soc., 78, 226-230.

Mason, P., 1994: Large eddy simulations: A critical review of the technique. Quart. J. Roy. Meteor. Soc., 120, 1-26.

Nicholls, S., 1984: The dynamics of stratocumulus: Aircraft observations and comparisons with a mixed layer model. Quart. J. Roy. Meteor. Soc., 110, 783-820.

— 1987: A model of drizzle growth in warm, turbulent, stratiform clouds. Quart. J. Roy. Meteor. Soc., 113, 1141-1170.

— of stratiform cloud sheets: Part I. Structure. Quart. J. Roy. Meteor. Soc., 112, 431-460.

Paluch, I. R., and D. H. Lenschow, 1991: Stratiform cloud formation in the marine boundary layer. J. Atmos. Sci., 48, 2141-2157.

Pincus, R., and M. B. Baker, 1994: Effect of precipitation on the albedo susceptibility of marine boundary layer clouds. Nature, 372, 250-252.

Pruppacher, H. R., and J. D. Klett, 1978: Microphysics of Clouds and Precipitation. D. Reidel, 714 pp.

Randall, D. A., and M. J. Suarez, 1984: On the dynamics of stratocumulus formation and dissipation. J. Atmos. Sci., 41, 30523057.

Schubert, W. H., J. S. Wakefield, E. J. Steiner, and S. K. Cox, 1979: Marine stratocumulus convection. Part I: Governing equations and horizontally homogeneous solutions. J. Atmos. Sci., 36, 1286-1307.

Stevens, B., 1996: On the dynamics of precipitating stratocumulus. Ph.D. thesis, Colorado State University, 140 pp. [Available from Colorado State University, Fort Collins, CO 80523.]

—, G. Feingold, W. R. Cotton, and R. L. Walko, 1996: Elements of the microphysical structure of numerically simulated nonprecipitating stratocumulus. J. Atmos. Sci., 53, 980-1007.

— W. R. Cotton, and G. Feingold, 1997: A critique of one and two-dimensional models of boundary layer clouds with detailed representations of droplet microphysics. Atmos. Res., in press.

Turton, J. D., and S. Nicholls, 1987: A study of the diurnal variation of stratocumulus using a multiple mixed layer model. Quart. J. Roy. Meteor. Soc., 113, 969-1009.

Tzivion, S., G. Feingold, and Z. Levin, 1987: An efficient numerical solution to the stochastic collection equation. J. Atmos. Sci., 44, 3139-3149.

Wang, S., and B. A. Albrecht, 1986: A stratocumulus model with an internal circulation. J. Atmos. Sci., 43, 2374-2390.

_ , and Q. Wang, 1994: Roles of drizzle in a one-dimensional thirdorder turbulence closure model of the nocturnal stratus-topped marine boundary layer. J. Atmos. Sci., 51, 1559-1576.

Wyant, M. C., C. S. Bretherton, H. A. Rand, and D. E. Stevens, 1997: Numerical simulations and a conceptual model of the stratocumulus to trade cumulus transition. J. Atmos. Sci., 54, 168192. 